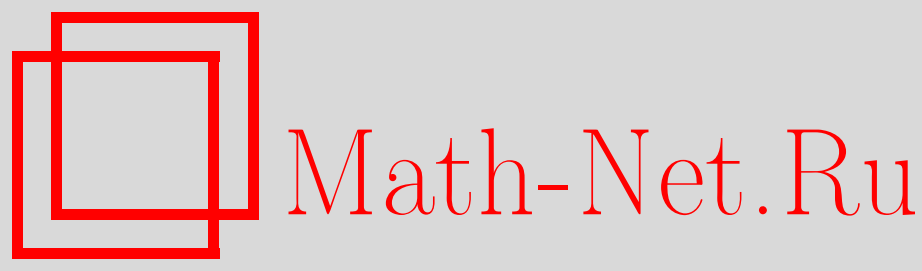

M. Deléglise, J.-L. Nicolas, Maximal Product of Primes Whose Sum is Bounded, Совр. пробл. матем., 2013, выпуск $17,82-112$

DOI: https://doi.org/10.4213/spm45

Использование Общероссийского математического портала Math-Net.Ru подразумевает, что вы прочитали и согласны с пользовательским соглашением http://www . mathnet.ru/rus/agreement

Параметры загрузки:

IP: 34.227 .88 .159

26 апреля 2023 г., 08:52:10

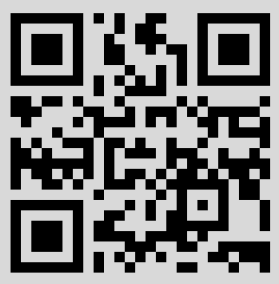




\title{
Maximal Product of Primes Whose Sum is Bounded
}

\author{
M. Deléglise, J.-L. Nicolas \\ Institut Camille Jordan, Université Claude Bernard Lyon 1, France
}

To the memory of A. A. Karatsuba, on the occasion of his 75 th birthday

\section{Introduction}

1.1. Function $\boldsymbol{h}(\boldsymbol{n})$. If $n \geqslant 2$ is an integer, let us define $h(n)$ as the greatest product of a family of primes $q_{1}<q_{2}<\cdots<q_{j}$ the sum of which does not exceed $n$.

Let $\ell$ be the additive function such that $\ell\left(p^{\alpha}\right)=p^{\alpha}$ for $p$ prime and $\alpha \geqslant 1$. In other words, if the standard factorization of $M$ into primes is $M=q_{1}^{\alpha_{1}} q_{2}^{\alpha_{2}} \cdots q_{j}^{\alpha_{j}}$, we have $\ell(M)=q_{1}^{\alpha_{1}}+q_{2}^{\alpha_{2}}+\cdots+q_{j}^{\alpha_{j}}$ and $\ell(1)=0$. Note that $\ell(n)$ is sequence A008475 in OEIS (cf. [1]). If $\mu$ denotes the Möbius function, $h(n)$ can also be defined as

$$
h(n)=\max _{\substack{\ell(M) \leqslant n \\ \mu(M) \neq 0}} M .
$$

Note that

$$
\ell(h(n)) \leqslant n .
$$

From the uniqueness of the factorization of $h(n)$ into primes, the maximum in (1.1) is attained at only one point. It is convenient to set

$$
h(0)=h(1)=1 \text {. }
$$

$(h(n))_{n \geqslant 1}$ is sequence A159685 of [1]. A table of the 50 first values of $h(n)$ s given at the end of Section 3. A larger table may be found on the authors's web sites [2], [3].

In [4], Landau has introduced the function $g(n)$ as the maximal order of an element in the symmetric group $\mathfrak{S}_{n}$; he has shown that

$$
g(n)=\max _{\ell(M) \leqslant n} M
$$

The introductions of [5] and [6] recall the main properties of Landau's function $g(n)$ which is mentioned as entry A002809 in [1]. From (1.1) and (1.3), it follows that

$$
h(n) \leqslant g(n), \quad n \geqslant 0 .
$$

In this article, we shall give some properties of $h(n)$ and describe an algorithm able to calculate $h(n)$ for any $n$ up to $10^{35}$.

1.2. Notation. We denote by $\mathbb{N}$ the set of non-negative integers.

The symbol $p$ will always denote a prime number.

For every arithmetic function $f: \mathbb{N} \rightarrow \mathbb{C}$, we define

$$
\pi_{f}(x)=\sum_{p \leqslant x, p \text { prime }} f(p) .
$$

Research supported by CNRS, Institut Camille Jordan, UMR 5208. 
In particular, for $f(n)=1$, we will note, as usual $\pi(x)=\pi_{1}(x)$ the number of primes up to $x$. For $f(n)=n$ we define

$$
\pi_{\mathrm{id}}(x)=\sum_{p \leqslant x, p \text { prime }} p .
$$

We denote by $p_{j}$ the $j$ th prime and we set $\sigma_{0}=0, N_{0}=1$ and, for $j \geqslant 1$,

$$
\sigma_{j}=\pi_{\mathrm{id}}\left(p_{j}\right)=p_{1}+p_{2}+\cdots+p_{j}, \quad N_{j}=p_{1} p_{2} \cdots p_{j}
$$

In Section 3, for all $j \geqslant 1$, we shall prove that $h\left(\sigma_{j}\right)=N_{j}$.

If $m$ is an integer, we denote by $(m)^{\star}$ the smallest prime $p$ satisfying $p \geqslant m$ and, if $m \geqslant 2$, by $\star(m)$ the largest prime $p$ satisfying $p \leqslant m$.

$P^{+}(m)\left(P^{-}(m)\right)$ will denote the largest (smallest) prime factor of $m \geqslant 2$. It is convenient to set $P^{+}(1)=-\infty$ and $P^{-}(1)=+\infty$.

$\omega(n)$ is the number of distinct prime factors of $n$ and $\Omega(n)$ the number of prime factors of $n$, counted with multiplicity. $\mu(n)$ is Möbius's function.

For $x>1$, we denote by $\log x$ the natural $\log$ arithm of $x$ and by $\log \log x=\log (\log x)$ the iterated logarithm of $x$.

$\mathrm{Li}$ is the integral logarithm defined for $x>1$ by

$$
\operatorname{Li}(x)=\lim _{\varepsilon \rightarrow 0, \varepsilon>0} \int_{0}^{1-\varepsilon}+\int_{1+\varepsilon}^{x} \frac{d t}{\log t}=\gamma+\log \log x+\sum_{n \geqslant 1} \frac{(\log x)^{n}}{n n !}
$$

where $\gamma$ is Euler's constant.

1.3. Functions $\boldsymbol{h}_{\boldsymbol{j}}(\boldsymbol{n})$. For $n \geqslant 0$, let $k=k(n)$ be the non-negative integer defined by

$$
\sigma_{k}=\pi_{\mathrm{id}}\left(p_{k}\right) \leqslant n<\pi_{\mathrm{id}}\left(p_{k+1}\right)=\sigma_{k+1} .
$$

It is the maximal number of prime factors of $h(n)$. For $0 \leqslant j \leqslant k=k(n)$, let us set

$$
h_{j}(n)=\max _{\substack{\ell(M) \leqslant n \\ \mu(M) \neq 0, \omega(M)=j}} M
$$

where $\omega(M)$ is the number of prime factors of $M$. For $n \geqslant 0$, we have

$$
h_{0}(n)=1
$$

while, for $n \geqslant 2$, we have

$$
h_{1}(n)={ }^{\star}(n) \geqslant 2 .
$$

Note that

$$
\ell\left(h_{j}(n)\right) \leqslant n
$$

In Section 6 , we prove that, for all $n$ 's, the sequence $h_{j}(n)$ is increasing on $j$, so that

$$
h(n)=h_{k}(n), \quad n \geqslant 0 .
$$

Our proof is not that simple. A possible reason is that this increasingness relies on the properties of the whole set of primes $\mathcal{P}$. Let $\mathcal{P}^{\prime}$ be a subset of $\mathcal{P}$ and $\mathcal{N}_{\mathcal{P}^{\prime}}$ the set of integers whose prime factors belong to $\mathcal{P}^{\prime}$. We may consider

$$
h_{j}\left(n, \mathcal{P}^{\prime}\right)=\max _{\substack{M \in \mathcal{N}_{\mathcal{P}^{\prime}}, \ell(M) \leqslant n \\ \mu(M) \neq 0, \omega(M)=j}} M
$$


By choosing $\mathcal{P}^{\prime}=\{2,3,11,13,17,19,23, \ldots\}=\mathcal{P} \backslash\{5,7\}$, we observe that

$$
h_{2}\left(24, \mathcal{P}^{\prime}\right)=11 \cdot 13=143>h_{3}\left(24, \mathcal{P}^{\prime}\right)=2 \cdot 3 \cdot 19=114 .
$$

In Section 4, we give an upper bound for $h_{j}(n)$ which will be useful in Section 6 where our proof of the increasingness of $h_{j}$ is given. In $(1.9), h_{j}(n)$ can be considered as the solution of a problem of optimization with prime variables. The upper bound of $h_{j}(n)$ is obtained by relaxing some constraints so that certain variables are no longer primes, but only integers.

1.4. Elementary computation of $\boldsymbol{h}(\boldsymbol{n})$ and $\boldsymbol{h}_{\boldsymbol{j}}(\boldsymbol{n})$. The naive algorithm described in [5] to compute $g(n)$ can be easily adapted to calculate $h(n)$ for $1 \leqslant n \leqslant N$. Note that, for the prime factors of $h(n)$, Corollary 3.1 below furnishes the upper bound

$$
P^{+}(h(n)) \leqslant p_{k(n)+1}+p_{k(n)+2} .
$$

It also can be adapted to compute $h_{j}(n)$. For $r \geqslant j \geqslant 1$ and $n \geqslant \sigma_{j}$, let us define

$$
h_{j}^{(r)}(n)=\max _{\substack{P^{+}(M) \leqslant p_{r}, \ell(M) \leqslant n \\ \mu(M) \neq 0, \omega(M)=j}} M .
$$

We have the induction relation

$$
h_{j}^{(r+1)}(n)=\max \left(h_{j}^{(r)}(n), p_{r+1} h_{j-1}^{(r)}\left(n-p_{r+1}\right)\right) .
$$

Indeed, either $p_{r+1}$ does not divide $h_{j}^{(r+1)}(n)$, and $h_{j}^{(r+1)}(n)=h_{j}^{(r)}(n)$ holds, or $p_{r+1}$ divides $h_{j}^{(r+1)}(n)$, and $h_{j}^{(r+1)}(n)=p_{r+1} h_{j-1}^{(r)}\left(n-p_{r+1}\right)$, which implies $n \geqslant p_{r+1}+\sigma_{j-1}$.

Moreover, if $p_{r} \geqslant n$, we have $h_{j}^{(r)}(n)=h_{j}(n), h_{r}^{(r)}(n)=N_{r}$ and $h_{1}^{(r)}(n)={ }^{\star}(n)$ for $n<p_{r}$ while, for $n \geqslant p_{r}, h_{1}^{(r)}(n)=p_{r}$ holds. So, we may write Algorithm 1, which has been used to calculate Table 1. The merging and pruning method described in [5; Sect. 2.2] can be used to improve the running time.

Algorithm 1. Computation of $h_{j}(n)$ for $2 \leqslant n \leqslant n \max$ and $1 \leqslant j \leqslant k(n)$.

Procedure ComputeHj(nmax)

$$
\begin{aligned}
& r=1 ; p=p_{r} ; k \max =k(\text { nmax }) ; \max =p_{k \max +1}+p_{k \max +2} \\
& \text { while } p \leqslant \max \text { do } \\
& \text { for } n \text { from } \sigma_{r} \text { to } n \max \text { do } \\
& \quad H[r, n]=N_{r} \\
& j \max =\min (r-1, k \max ) \\
& \text { for } j \text { from } j \max \text { by }-1 \text { to } 2 \text { do } \\
& \quad \text { for } n \text { from } \max \text { by }-1 \text { to } p+\sigma_{j-1} \text { do } \\
& \qquad H[j, n]=\max (H[j, n], p * H[j-1, n-p]) \\
& \text { for } n \text { from } p \text { to } n \max \\
& \quad H[1, n]=p \\
& r=r+1 ; p=p_{r} .
\end{aligned}
$$

In Section 8, a more sophisticated algorithm to calculate $h(n)$ is given. It is based on a fast method to compute $\pi_{\text {id }}(x)$, which is explained in Section 7 .

\section{Some lemmas}

Lemma 2.1. If $m \geqslant 2$ is an integer, let us denote by $(m)^{\star}\left(^{\star}(m)\right)$ the smallest (largest) prime $p$ satisfying $p \geqslant m(p \leqslant m)$. Then

$$
(m)^{\star} \leqslant \frac{11}{8} m \quad \text { and } \quad \star(m) \geqslant \frac{7}{10} m
$$

hold. 
Proof. We use the result of [7]: for $x \geqslant 396738$, the interval $\left[x, x+x /\left(25 \log ^{2} x\right)\right]$ contains a prime number. As 396833 is prime, we deduce that, for $p_{i} \geqslant 396833$,

$$
\frac{p_{i+1}}{p_{i}} \leqslant 1+\frac{1}{25 \log ^{2} p_{i}} \leqslant 1+\frac{1}{25 \log ^{2} 396833}<1.00025<\frac{11}{8}<\frac{10}{7} .
$$

If $m$ is prime, $(m)^{\star}={ }^{\star}(m)=m$ holds, while, if $m$ is not prime, we define $p_{i}$ by $p_{i}<m<p_{i+1}$; we have $(m)^{\star}=p_{i+1},{ }^{\star}(m)=p_{i}$,

$$
\frac{(m)^{\star}}{m} \leqslant \frac{p_{i+1}}{p_{i}+1}<\frac{p_{i+1}}{p_{i}}, \quad \frac{\star(m)}{m} \geqslant \frac{p_{i}}{p_{i+1}-1}>\frac{p_{i}}{p_{i+1}}
$$

and, if $p_{i} \geqslant 396833$, the result follows from (2.1). Finally, it remains to check that

$$
\frac{p_{i+1}}{p_{i}+1} \leqslant \frac{11}{8} \quad \text { and } \quad \frac{p_{i}}{p_{i+1}-1} \geqslant \frac{7}{10}
$$

hold for all $p_{i}$ 's satisfying $2 \leqslant p_{i}<396833$.

LEMMA 2.2. Let $p<p^{\prime}$ be two primes. There exists a third prime $p^{\prime \prime}$ satisfying

$$
p+p^{\prime} \leqslant p^{\prime \prime} \leqslant p p^{\prime}-p+1
$$

Proof. Let us show that $p^{\prime \prime}=\left(p+p^{\prime}\right)^{\star}$ satisfies (2.2). By Lemma 2.1, it suffices to prove that $(11 / 8)\left(p+p^{\prime}\right) \leqslant p p^{\prime}-p+1$, i.e.

$$
p p^{\prime}\left(8-\frac{11}{p}-\frac{19}{p^{\prime}}+\frac{8}{p p^{\prime}}\right) \geqslant 0
$$

If $p \geqslant 3$ and $p^{\prime} \geqslant 5$, we have $11 / p+19 / p^{\prime} \leqslant 11 / 3+19 / 5<8$ and (2.3) holds. Similarly, if $p=2$ and $p^{\prime} \geqslant 11$, the inequality $11 / p+19 / p^{\prime} \leqslant 11 / 2+19 / 11<8$ implies $(2.3)$. In the three remaining cases, $p=2$ and $p^{\prime} \in\{3,5,7\}$, it is easy to check that $p^{\prime \prime}=\left(p+p^{\prime}\right)^{\star}$ satisfies $(2.2)$.

LEMMA 2.3. Let $p$ and $p^{\prime}$ be two prime numbers satisfying $3 \leqslant p<p^{\prime}$ and $p p^{\prime} \neq 15$. There exists a prime $p^{\prime \prime}$ such that

$$
p+p^{\prime} \leqslant p^{\prime \prime} \leqslant \frac{5}{6} p p^{\prime}-p .
$$

Proof. The proof is similar to the one of the preceding lemma. From Lemma 2.1, to show that $p^{\prime \prime}=\left(p+p^{\prime}\right)^{\star}$ satisfies $(2.4)$, it suffices to show that $(11 / 8)\left(p+p^{\prime}\right) \leqslant(5 / 6) p p^{\prime}-p$, i.e. $33 / p+57 / p^{\prime} \leqslant 20$, which evidently holds for $p \geqslant 3$ and $p^{\prime} \geqslant 7$.

LEMMA 2.4. For all $i \geqslant 2$, the following inequality

$$
p_{i}+p_{i-1} \leqslant p_{2 i-1}
$$

holds. Moreover, let $b$ be a positive integer; there exists a positive integer $i_{0}=i_{0}(b)$ such that we have

$$
p_{i}+p_{i-1}<p_{2 i-b} \quad \text { for } \quad i \geqslant i_{0}(b) .
$$

The table below gives some values of $i_{0}(b)$

\begin{tabular}{|c|ccccccccccccccc|}
\hline$b$ & 1 & 2 & 3 & 4 & 5 & 6 & 7 & 8 & 9 & 10 & 12 & 13 & 18 & 30 & 3675 \\
\hline$i_{0}$ & 3 & 4 & 7 & 8 & 18 & 19 & 27 & 28 & 36 & 39 & 50 & 53 & 85 & 149 & 33127 \\
\hline
\end{tabular}


Proof. We start from the two inequalities

$$
\begin{array}{ll}
p_{i} \leqslant i(\log i+\log \log i-\alpha), \quad \alpha=0.9484, & i \geqslant 39017, \\
p_{i} \geqslant i(\log i+\log \log i-1), & i \geqslant 2,
\end{array}
$$

which can be found in [8]. From (2.7), it follows that

$$
p_{i-1}+p_{i} \leqslant(2 i-1)(\log i+\log \log i-\alpha), \quad i \geqslant 39018,
$$

while, if $i \geqslant \max (2, b)$, which implies $2 i-b \geqslant 2$ and $i \geqslant b,(2.8)$ gives

$$
p_{2 i-b} \geqslant(2 i-b)\left(\log i+\log 2+\log \frac{2 i-b}{2 i}+\log \log i-1\right) .
$$

By using the inequality $\log t \leqslant t-1$, we get

$$
\log \frac{2 i-b}{2 i}=-\log \frac{2 i}{2 i-b} \geqslant-\left(\frac{2 i}{2 i-b}-1\right)=-\frac{b}{2 i-b}
$$

and (2.10) yields

$$
p_{2 i-b} \geqslant(2 i-b)(\log i+\log \log i+\log 2-1)-b .
$$

Under the condition

$$
i \geqslant \max (39018, b)
$$

the substraction of (2.9) from (2.11) gives

$$
\begin{aligned}
p_{2 i-b}-p_{i-1}-p_{i} & \geqslant(\log i+\log \log i+\log 2)(1-b)+2 i(\log 2-1+\alpha)-\log 2-\alpha \\
& >(\log i+\log \log i+\log 2)\left[\frac{1.283 i-1.642}{\log i+\log \log i+\log 2}-(b-1)\right] .
\end{aligned}
$$

Now, the two functions $t \mapsto t /(\log t+\log \log t+\log 2)$ and $t \mapsto-1 /(\log t+\log \log t+\log 2)$ are increasing for $t \geqslant e^{2}$; choosing $i_{1}=39018$ and

$$
b=\left\lfloor 1+\frac{1.283 i_{1}-1.642}{\log i_{1}+\log \log i_{1}+\log 2}\right\rfloor=\lfloor 3675.52 \ldots\rfloor=3675
$$

shows that, for $i \geqslant i_{1},(2.12)$ is satisfied and that in (2.13), the bracket is positive. Therefore, (2.13) proves $p_{i}+p_{i-1}<p_{2 i-3675}$ for $i \geqslant i_{1}=39018$.

To determine the entries of the table, for all $i$ 's up to 39018 , we have calculated $b_{i}=2 i-1-$ $\pi\left(p_{i}+p_{i-1}\right)$ which is the smallest integer such that $p_{i-1}+p_{i}<p_{2 i-b_{i}}$. Further, for each $b$ in the table, we have determined $i_{0}(b)$ which is the smallest integer $i_{0}$ such that, for $i_{0}(b) \leqslant i \leqslant 39018$, $b_{i} \geqslant b$ holds.

As $i_{0}(1)=3$, for all $i \geqslant 3, p_{i}+p_{i-1}<p_{2 i-1}$ holds. So, (2.5) follows from $p_{2}+p_{1}=3+2=5=p_{3}$.

LEMMA 2.5. Under Riemann hypothesis, for all $x \geqslant 41$ we have

$$
\left|\pi_{\mathrm{id}}(x)-\operatorname{Li}\left(x^{2}\right)\right| \leqslant \frac{5}{24 \pi} x^{3 / 2} \log x .
$$

Proof. Let us define $r(x)$ by $\pi(x)=\operatorname{Li}(x)+r(x)$ and assume the Riemann hypothesis. Then (cf. $[9 ;(6.18)])$ :

$$
|r(x)|=|\pi(x)-\operatorname{Li}(x)| \leqslant \frac{1}{8 \pi} \sqrt{x} \log x \quad \text { for } \quad x \geqslant 2657 \text {. }
$$


Let us denote $x_{0}=2657$. Then, from (1.6), Stieltjes's integral gives:

$$
\begin{aligned}
\pi_{\mathrm{id}}(x) & =\pi_{\mathrm{id}}\left(x_{0}\right)+\int_{x_{0}^{-}}^{x} t d[\pi(t)]=\pi_{\mathrm{id}}\left(x_{0}\right)+\int_{x_{0}}^{x} t d(\operatorname{Li}(t))+\int_{x_{0}^{-}}^{x} t d[r(t)] \\
& =\pi_{\mathrm{id}}\left(x_{0}\right)+\operatorname{Li}\left(x^{2}\right)-\operatorname{Li}\left(x_{0}^{2}\right)+\left.\operatorname{tr}(t)\right|_{x_{0}} ^{x}-\int_{x_{0}}^{x} r(t) d t
\end{aligned}
$$

With (2.16), it comes

$$
\left|\pi_{\text {id }}(x)-\operatorname{Li}\left(x^{2}\right)\right| \leqslant\left|\pi_{\text {id }}\left(x_{0}\right)-\operatorname{Li}\left(x_{0}^{2}\right)-x_{0} r\left(x_{0}\right)\right|+\frac{x^{3 / 2} \log x}{8 \pi}+\int_{x_{0}}^{x} \frac{\sqrt{t} \log t}{8 \pi} d t
$$

and, using

$$
\begin{gathered}
\int \sqrt{t} \log t=\frac{2}{3} t^{3 / 2}\left(\log t-\frac{2}{3}\right), \\
\left|\pi_{\mathrm{id}}(x)-\operatorname{Li}\left(x^{2}\right)\right| \\
\quad \leqslant \frac{5}{24 \pi} x^{3 / 2} \log x-\frac{1}{18 \pi} x^{3 / 2}+\left|\pi_{\mathrm{id}}\left(x_{0}\right)-\operatorname{Li}\left(x_{0}^{2}\right)-x_{0} r\left(x_{0}\right)\right|-\frac{1}{12 \pi} x_{0}^{3 / 2} \log x_{0}+\frac{1}{18 \pi} x_{0}^{3 / 2} .
\end{gathered}
$$

The computation of

$$
\begin{aligned}
r\left(x_{0}\right)=\pi\left(x_{0}\right)-\operatorname{Li}\left(x_{0}\right) & =384-399.59681 \ldots=-15.59681 \ldots, \\
& \pi_{\mathrm{id}}\left(x_{0}\right)-\operatorname{Li}\left(x_{0}^{2}\right)=464653-480610.2863 \ldots=-15957.2863 \ldots
\end{aligned}
$$

and (2.17) imply for $x \geqslant x_{0}$,

$$
\left|\pi_{\mathrm{id}}(x)-\operatorname{Li}\left(x^{2}\right)\right| \leqslant \frac{5}{24 \pi} x^{3 / 2} \log x-\frac{1}{18 \pi} x^{3 / 2}-740.023 \ldots \leqslant \frac{5}{24 \pi} x^{3 / 2} \log x .
$$

which proves $(2.15)$ for $x \geqslant x_{0}=2657$. It remains to check $(2.15)$ for $41 \leqslant x \leqslant 2657$; by setting

$$
f_{1}(x)=\operatorname{Li}\left(x^{2}\right)-\frac{5}{24 \pi} x^{3 / 2} \log x, \quad f_{2}(x)=\operatorname{Li}\left(x^{2}\right)+\frac{5}{24 \pi} x^{3 / 2} \log x,
$$

it is equivalent to check

$$
f_{1}(x) \leqslant \pi_{\text {id }}(x) \leqslant f_{2}(x)
$$

for $41 \leqslant x \leqslant 2657$. One remarks that $f_{1}$ and $f_{2}$ are increasing for $x \geqslant 2$. Therefore, to prove (2.18), it suffices to check that for every prime $p$ satisfying $41 \leqslant p \leqslant 2657$ we have $f_{1}\left(p^{\prime}\right) \leqslant \pi_{\text {id }}(x) \leqslant f_{2}(p)$ where $p^{\prime}$ is the prime following $p$.

Note that, in the range $[2,2657], \pi_{\mathrm{id}}(x)-\operatorname{Li}\left(x^{2}\right)$ has several changes of sign, the smallest one being for $x=110.35 \ldots$.

Lemma 2.6. Let $z$ and $u$ be two real numbers satisfying $z \geqslant 1$ and $\sqrt{z} \leqslant u \leqslant z$. Suppose that we have precomputed the tables prime, piftab and pi. The first two tables are indexed by the integers $k, 0 \leqslant k \leqslant \pi(u)$, and the third one by the integers $t, 0 \leqslant t \leqslant u$,

- $\operatorname{prime}[k]$ contains $p_{k}\left(p_{0}=1\right)$;

- piftab $[k]$ contains $\pi_{f}\left(p_{k}\right)$;

- $\mathrm{pi}[t]$ contains $\pi(t)$.

Then the sum

$$
\sum_{\sqrt{z}<q \leqslant u, q \text { prime }} f(q) \pi_{f}\left(\frac{z}{q}\right)
$$

may be computed in $O(\sqrt{z} / \log z)$ time. 
Proof. For $q>\sqrt{z}, z / q$ belongs to $[1, \sqrt{z})$. The number of primes in this interval is $O(\sqrt{z} / \log z)$, thus the number of values of $\pi_{f}(z / q)$ is $O(\sqrt{z} / \log z)$. We group the $q$ 's for which $\pi_{f}(z / q)$ takes the same value. Algorithm 2 carries out this computation.

Algorithm 2. Computation of the $\operatorname{sum}(2.19)$ in $O(\sqrt{z} / \log z)$ time.

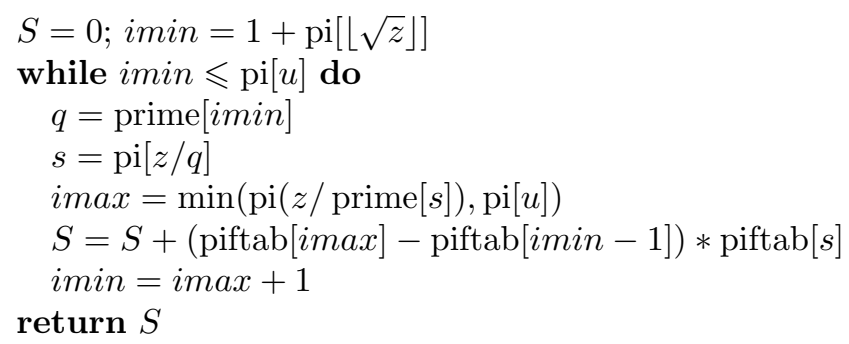

Let us give some words to convince of the correctness of algorithm 2: let us note $s=\pi(z / q)$. Then $p_{s}$ is the largest prime $\leqslant z / q$. For $q^{\prime}$ prime, $q^{\prime} \geqslant q$, we have $\pi_{f}\left(z / q^{\prime}\right)=\pi_{f}\left(p_{s}\right)=\operatorname{piftab}[s]$ if and only if $z / q^{\prime} \geqslant p_{s}$ i.e. $q^{\prime} \leqslant z / p_{s}$, in other terms, $\pi\left(q^{\prime}\right) \leqslant \pi\left(z / p_{s}\right)$. Thus the largest prime $q^{\prime}$ in the range $[q, u]$ such that $\pi_{f}\left(z / q^{\prime}\right)=\pi_{f}\left(p_{s}\right)$ is $p_{i}$ where $i=\min \left(\pi\left(z / p_{s}\right), \pi(u)\right)$.

\section{First results}

Proposition 3.1. Let $j$ be a positive integer and $\sigma_{j}$ and $N_{j}$ be defined by (1.7). We have

$$
h\left(\sigma_{j}\right)=N_{j} .
$$

Proof. It is easy to see that $h\left(\sigma_{1}\right)=h(2)=2=N_{1}$ and $h\left(\sigma_{2}\right)=h(5)=6=N_{2}$. Now, we may suppose that $j \geqslant 3$, i.e. $p_{j} \geqslant 5$ and we set $\rho=p_{j} / \log p_{j}$. The function $t \mapsto t / \log t$ is increasing for $t \geqslant e$ and, since $2 / \log 2<5 / \log 5$, we have, for $1 \leqslant i<j, p_{i} / \log p_{i}<\rho$ and for $i>j$, $p_{i} / \log p_{i}>\rho$; in other words, $i-j$ and $p_{i} / \log p_{i}-\rho$ have the same sign.

Let $M$ be a product of $r$ distinct primes, $M=Q_{1} Q_{2} \cdots Q_{r}$, with $r \geqslant 0$. After a possible simplification by $s$ primes, $0 \leqslant s \leqslant \min (j, r)$, we may write

$$
\frac{M}{N_{j}}=\frac{p_{j_{1}} p_{j_{2}} \cdots p_{j_{u}}}{p_{k_{1}} p_{k_{2}} \cdots p_{k_{v}}}
$$

with $u=r-s, v=j-s$ and

$$
p_{k_{1}}<p_{k_{2}}<\cdots<p_{k_{v}} \leqslant p_{j}<p_{j_{1}}<p_{j_{2}}<\cdots<p_{j_{u}} .
$$

Let $f(M)=\ell(M)-\rho \log M$. From the definition of $\ell$, the function $f$ is additive and we have

$$
f(M)-f\left(N_{j}\right)=\sum_{i=1}^{u}\left(p_{j_{i}}-\rho \log p_{j_{i}}\right)-\sum_{i=1}^{v}\left(p_{k_{i}}-\rho \log p_{k_{i}}\right) \geqslant 0
$$

since each term of the first sum is non-negative while, in the second sum, each term is non-positive.

From (1.1), since $\ell\left(N_{j}\right)=\sigma_{j}$, in order to prove that $h\left(\sigma_{j}\right)=N_{j}$, we must show that, for all squarefree number $M$ satisfying $\ell(M) \leqslant \sigma_{j}=\ell\left(N_{j}\right)$, we have $M \leqslant N_{j}$. But, for such an $M,(3.1)$ yields

$$
f(M)=\ell(M)-\rho \log M \geqslant f\left(N_{j}\right)=\ell\left(N_{j}\right)-\rho \log N_{j}=\sigma_{j}-\rho \log N_{j}
$$

whence

$$
\frac{M}{N_{j}} \leqslant \exp \left(\frac{\ell(M)-\sigma_{j}}{\rho}\right) \leqslant 1,
$$

which completes the proof of Proposition 3.1. 
Proposition 3.2. Let $r$ and $j$ be two positive integers and $\sigma_{j}, N_{j}$ and $h_{j}$ be defined by (1.7) and (1.9). We have

$$
h_{j}\left(\sigma_{j+r}-\sigma_{r}\right)=\frac{N_{j+r}}{N_{r}}=p_{r+1} p_{r+2} \cdots p_{r+j} .
$$

Moreover, if $n \geqslant \sigma_{j+r}-\sigma_{r}$ we have

$$
\ell\left(h_{j}(n)\right) \geqslant \sigma_{j+r}-\sigma_{r} .
$$

Proof. The proof is similar to the one of Proposition 3.1. Let us set

$$
\rho=\frac{p_{j+r}-p_{r}}{\log \left(p_{j+r} / p_{r}\right)} \quad \text { and } \quad \rho^{\prime}=\rho \log p_{r}-p_{r}
$$

Since, for $t \neq 1,(t-1) / t<\log t<t-1$ holds, we have $p_{j+r}>\rho>p_{r} \geqslant 2$. For a squarefree number $M$, we consider the additive function

$$
f(M)=\ell(M)-\rho \log M+\rho^{\prime} \omega(M)=\sum_{p \mid M} f(p)=\sum_{p \mid M}\left(p-\rho \log p+\rho^{\prime}\right) .
$$

We will prove that $f$ attains its minimum in $N=N_{j+r} / N_{r}$. From (3.4), it follows that $f\left(p_{j+r}\right)=$ $f\left(p_{r}\right)=0$ and the study of the function $t \mapsto t-\rho \log t+\rho^{\prime}$ shows that

$$
\begin{cases}f(p)>0 & \text { for } \quad p<p_{r} \text { or } p>p_{j+r} \\ f(p)<0 & \text { for } \quad p_{r}<p<p_{j+r} \\ f(p)=0 & \text { for } \quad p=p_{r} \text { or } p=p_{j+r}\end{cases}
$$

Therefore, we have

$$
f(M)-f(N)=\sum_{\substack{p \mid M \\ p<p_{r} \text { or } p>p_{j+r}}} f(p)-\sum_{\substack{p \nmid M \\ p_{r}<p<p_{j+r}}} f(p) \geqslant 0 .
$$

From (1.9), we have to show that, for any squarefree integer $M$ satisfying $\ell(M) \leqslant \sigma_{j+r}-\sigma_{r}=\ell(N)$ and $\omega(M)=j=\omega(N)$, we have $M \leqslant N$. For such an $M,(3.5)$ gives

$$
\ell(M)-\rho \log M+\rho^{\prime} \omega(M) \geqslant \ell(N)-\rho \log N+\rho^{\prime} \omega(N)
$$

yielding

$$
\frac{M}{N} \leqslant \exp \left(\frac{\ell(M)-\ell(N)}{\rho}\right) \leqslant 1,
$$

which, together with $\ell(N)=\sigma_{j+r}-\sigma_{r}$, proves $(3.2)$.

To prove (3.3), first, from (1.9), we observe that $h_{j}(n) \geqslant N=N_{j+r} / N_{r}$. Setting $M=h_{j}(n)$ in (3.5) and noting that $\omega(M)=\omega(N)=j$, we see that

$$
\ell(M) \geqslant \ell(N)+\rho \log \frac{M}{N} \geqslant \ell(N)=\sigma_{j+r}-\sigma_{r}
$$

which proves (3.3).

Proposition 3.3. Let $n \geqslant 2$ be an integer and $p<p^{\prime}$ two prime numbers which do not divide $h(n)$. Then the largest prime divisor $P^{+}(h(n))$ of $h(n)$ satisfies

$$
P^{+}(h(n))<p+p^{\prime}
$$


Proof. Let us assume that the set of prime factors of $h(n)$ not smaller than $p+p^{\prime}$ is not empty and let $q \geqslant p+p^{\prime}$ be its smallest element.

If $q<p p^{\prime}$, by setting $M=\left(p p^{\prime} / q\right) h(n)$, we have by (1.2)

$$
\ell(M)=p+p^{\prime}-q+\ell(h(n)) \leqslant \ell(h(n)) \leqslant n
$$

and thus, from (1.1),

$$
h(n) \geqslant M=\frac{p p^{\prime}}{q} h(n),
$$

in contradiction with $q<p p^{\prime}$.

If $q>p p^{\prime}$, i.e. $q \geqslant p p^{\prime}+1$, by Lemma 2.2 , the interval $\left[p+p^{\prime}, q-p\right]$ contains a prime; thus the prime $p^{\prime \prime}={ }^{\star}(q-p)$ satisfies $p+p^{\prime} \leqslant p^{\prime \prime} \leqslant q-p<q$ and, from the definition of $q$, $p^{\prime \prime}$ does not divide $h(n)$. By Lemma 2.1, $p^{\prime \prime}={ }^{\star}(q-p) \geqslant(7 / 10)(q-p)$ holds, whence

$$
q \leqslant \frac{10}{7} p^{\prime \prime}+p=\frac{p p^{\prime \prime}}{7}\left(\frac{10}{p}+\frac{7}{p^{\prime \prime}}\right) .
$$

We have $p \geqslant 2, p^{\prime} \geqslant 3$ and $p^{\prime \prime} \geqslant p+p^{\prime} \geqslant 5$, so that $10 / p+7 / p^{\prime \prime} \leqslant 10 / 2+7 / 5<7$, yielding $q<p p^{\prime \prime}$. By considering $M=\left(p p^{\prime \prime} / q\right) h(n)$, as in (3.6), we get

$$
h(n) \geqslant M=\frac{p p^{\prime \prime}}{q} h(n)>h(n),
$$

a contradiction.

Corollary 3.1. If $k=k(n)$ is defined by (1.8), the largest prime factor of $h(n)$ satisfies

$$
P^{+}(h(n))<p_{k+1}+p_{k+2} \text {. }
$$

Proof. The number of prime factors of $h(n)$ does not exceed $k$, so that, among $p_{1}, p_{2}, \ldots, p_{k+2}$ there are certainly two prime numbers $p$ and $p^{\prime}$ not dividing $h(n)$. By applying Proposition 3.3, we get $P^{+}(h(n))<p+p^{\prime} \leqslant p_{k+1}+p_{k+2}$.

Proposition 3.4. Let $n \geqslant 5$ be an integer, $k \geqslant 2$ be defined by (1.8) and $j$ an integer satisfying $2 \leqslant j \leqslant k$. Let us supose that there exists two prime numbers, $p, p^{\prime}$ not dividing $h_{j-1}(n)$, and satisfying $3 \leqslant p<p^{\prime}$ and $P^{+}\left(h_{j-1}(n)\right) \geqslant p+p^{\prime}$ where $P^{+}\left(h_{j-1}(n)\right)$ is the largest prime divisor of $h_{j-1}(n)$. Then the inequality

$$
h_{j}(n)>\frac{6}{5} h_{j-1}(n)
$$

holds.

Proof. Let us consider two cases.

Case 1: $p p^{\prime}>15$. Let us denote by $q \leqslant P^{+}\left(h_{j-1}(n)\right)$ the smallest prime dividing $h_{j-1}(n)$ and satisfying $p+p^{\prime} \leqslant q$.

If $q<(5 / 6) p p^{\prime}$, we set $M=\left(p p^{\prime} / q\right) h_{j-1}(n)$; we have $\omega(M)=j$ and $\ell(M)=p+p^{\prime}-q+$ $\ell\left(h_{j-1}(n)\right) \leqslant \ell\left(h_{j-1}(n)\right)$ so that, from $(1.12), \ell(M) \leqslant n$ holds and (1.9) yields

$$
h_{j}(n) \geqslant M>\frac{6}{5} h_{j-1}(n)
$$

as required.

If $q \geqslant(5 / 6) p p^{\prime}$, we set $p^{\prime \prime}={ }^{\star}(q-p)$; from Lemma $2.3, p+p^{\prime} \leqslant p^{\prime \prime} \leqslant q-p<q$ holds, and, from the definition of $q, p^{\prime \prime}$ does not divide $h_{j-1}(n)$. 
By Lemma 2.1, we get $p^{\prime \prime}={ }^{\star}(q-p) \geqslant(7 / 10)(q-p)$, which implies

$$
q \leqslant \frac{10}{7} p^{\prime \prime}+p=\frac{p p^{\prime \prime}}{7}\left(\frac{10}{p}+\frac{7}{p^{\prime \prime}}\right)
$$

But $p \geqslant 3, p^{\prime} \geqslant 7, p^{\prime \prime} \geqslant p+p^{\prime} \geqslant 10$, thus $p^{\prime \prime} \geqslant 11$, and $10 / p+7 / p^{\prime \prime} \leqslant 10 / 3+7 / 11<35 / 6$, yielding $q<(5 / 6) p p^{\prime \prime}$. By setting $M=\left(p p^{\prime \prime} / q\right) h_{j-1}(n)$, as in $(3.7)$, we get $h_{j}(n) \geqslant M>(6 / 5) h_{j-1}(n)$.

Case 2: $p=3, p^{\prime}=5$.

If $P^{+}\left(h_{j-1}(n)\right) \leqslant 13$, which implies $n \leqslant \pi_{\text {id }}(13)=41$, examining Table 1 shows that, for $n \leqslant 41$, we have $h_{j}(n) \geqslant(6 / 5) h_{j-1}(n)$ with equality if and only if $h_{j-1}(n)=5,35,385$ or 5005 .

Table 1: Table of $h_{j}(n)$ and $h(n)$

\begin{tabular}{|c|c|c|c|c|c|c|}
\hline$n$ & $h_{1}$ & $h_{2}$ & $h_{3}$ & $h_{4}$ & $h_{5} \quad h_{6}$ & $h$ \\
\hline 2 & 2 & & & & & 2 \\
\hline 3 & 3 & & & & & 3 \\
\hline 4 & 3 & & & & & 3 \\
\hline 5 & 5 & 6 & & & & 6 \\
\hline 6 & 5 & 6 & & & & 6 \\
\hline 7 & 7 & 10 & & & & 10 \\
\hline 8 & 7 & 15 & & & & 15 \\
\hline 9 & 7 & 15 & & & & 15 \\
\hline 10 & 7 & 21 & 30 & & & 30 \\
\hline 11 & 11 & 21 & 30 & & & 30 \\
\hline 12 & 11 & 35 & 42 & & & 42 \\
\hline 13 & 13 & 35 & 42 & & & 42 \\
\hline 14 & 13 & 35 & 70 & & & 70 \\
\hline 15 & 13 & 35 & 105 & & & 105 \\
\hline 16 & 13 & 55 & 105 & & & 105 \\
\hline 17 & 17 & 55 & 105 & 210 & & 210 \\
\hline 18 & 17 & 77 & 110 & 210 & & 210 \\
\hline 19 & 19 & 77 & 165 & 210 & & 210 \\
\hline 20 & 19 & 91 & 165 & 210 & & 210 \\
\hline 21 & 19 & 91 & 231 & 330 & & 330 \\
\hline 22 & 19 & 91 & 231 & 330 & & 330 \\
\hline 23 & 23 & 91 & 385 & 462 & & 462 \\
\hline 24 & 23 & 143 & 385 & 462 & & 462 \\
\hline 25 & 23 & 143 & 455 & 770 & & 770 \\
\hline 26 & 23 & 143 & 455 & 1155 & & 1155 \\
\hline 27 & 23 & 143 & 455 & 1155 & & 1155 \\
\hline 28 & 23 & 187 & 455 & 1365 & 2310 & 2310 \\
\hline 29 & 29 & 187 & 715 & 1365 & 2310 & 2310 \\
\hline 30 & 29 & 221 & 715 & 1365 & 2730 & 2730 \\
\hline 31 & 31 & 221 & 1001 & 1430 & 2730 & 2730 \\
\hline 32 & 31 & 247 & 1001 & 2145 & 2730 & 2730 \\
\hline 33 & 31 & 247 & 1001 & 2145 & 2730 & 2730 \\
\hline 34 & 31 & 253 & 1001 & 3003 & 4290 & 4290 \\
\hline 35 & 31 & 253 & 1309 & 3003 & 4290 & 4290 \\
\hline 36 & 31 & 323 & 1309 & 5005 & 6006 & 6006 \\
\hline 37 & 37 & 323 & 1547 & 5005 & 6006 & 6006 \\
\hline 38 & 37 & 323 & 1547 & 5005 & 10010 & 10010 \\
\hline
\end{tabular}




\begin{tabular}{|r|rrrrrr|r|}
\hline$n$ & $h_{1}$ & $h_{2}$ & $h_{3}$ & $h_{4}$ & $h_{5}$ & $h_{6}$ & $h$ \\
\hline 39 & 37 & 323 & 1729 & 5005 & 15015 & & 15015 \\
40 & 37 & 391 & 1729 & 6545 & 15015 & & 15015 \\
41 & 41 & 391 & 2431 & 6545 & 15015 & 30030 & 30030 \\
42 & 41 & 437 & 2431 & 7735 & 15015 & 30030 & 30030 \\
43 & 43 & 437 & 2717 & 7735 & 19635 & 30030 & 30030 \\
44 & 43 & 437 & 2717 & 8645 & 19635 & 30030 & 30030 \\
45 & 43 & 437 & 2717 & 8645 & 23205 & 39270 & 39270 \\
46 & 43 & 493 & 2717 & 12155 & 23205 & 39270 & 39270 \\
47 & 47 & 493 & 3553 & 12155 & 25935 & 46410 & 46410 \\
48 & 47 & 551 & 3553 & 17017 & 25935 & 46410 & 46410 \\
49 & 47 & 551 & 4199 & 17017 & 36465 & 51870 & 51870 \\
50 & 47 & 589 & 4199 & 19019 & 36465 & 51870 & 51870 \\
\hline
\end{tabular}

If $P^{+}\left(h_{j-1}(n)\right) \geqslant 17$, and 11 does not divide $P^{+}\left(h_{j-1}(n)\right)$, then we apply case 1 with $p=3$, $p^{\prime}=11$, while, if 11 divides $P^{+}\left(h_{j-1}(n)\right)$,

$$
h_{j}(n) \geqslant \frac{3 \cdot 5}{11} h_{j-1}(n)>\frac{6}{5} h_{j-1}(n)
$$

holds.

\section{Bounding $h_{j}(n)$}

Proposition 4.1. Let $j \geqslant 1$ and $n \geqslant \sigma_{j}$ (where $\sigma_{j}$ has been introduced in (1.7)) be two integers; we define $r \geqslant 0$ by

$$
\sigma_{j+r}-\sigma_{r} \leqslant n<\sigma_{j+r+1}-\sigma_{r+1}
$$

and $n^{\prime}$ by

$$
0 \leqslant n^{\prime}=n-\left(\sigma_{j+r}-\sigma_{r}\right)<p_{j+r+1}-p_{r+1}
$$

Then we have

$$
h_{j}(n) \leqslant p_{r+1} p_{r+2} \cdots p_{r+j} \frac{p_{j+r+1}}{p_{j+r+1}-n^{\prime}}=\frac{N_{j+r+1}}{N_{r}\left(p_{j+r+1}-n^{\prime}\right)} .
$$

Proof. From its definition (1.9), $h_{j}(n)$ is a product of $j$ primes. Let us denote by $A_{1}, A_{2}, \ldots, A_{u}$ (with $0 \leqslant u \leqslant j$ ) its prime factors exceeding $p_{j+r+1}$ and by $B_{1}, B_{2}, \ldots, B_{r+1+u}$ the primes $\leqslant p_{j+r+1}$ and not dividing $h_{j}(n)$; we have

$$
h_{j}(n)=\frac{N_{j+r+1} A_{1} A_{2} \cdots A_{u}}{B_{1} B_{2} \cdots B_{r+1+u}}
$$

(where the product $A_{1} A_{2} \cdots A_{u}$ should be replaced by 1 when $u=0$ ) and

$$
2 \leqslant B_{1}<\cdots<B_{r+1+u} \leqslant p_{j+r+1}<p_{j+r+2} \leqslant A_{1}<\cdots<A_{u} .
$$

Further, let us introduce $\nu=\ell\left(h_{j}(n)\right)$; by (1.12) and (4.1), we have

$$
\nu \leqslant n<\sigma_{j+r+1}-\sigma_{r+1}
$$

and it follows from Proposition 3.2, (3.3), that

$$
\nu=\ell\left(h_{j}(n)\right) \geqslant \sigma_{j+r}-\sigma_{r} .
$$

Moreover, (4.4) implies

$$
\nu=\ell\left(h_{j}(n)\right)=\sigma_{j+r+1}-\sigma_{r}+\sum_{i=1}^{u}\left(A_{i}-B_{r+1+i}\right)-\sum_{i=1}^{r}\left(B_{i}-p_{i}\right)-B_{r+1} .
$$


Now, we consider the optimization problem (where $\nu, r, u, A_{1}, A_{2}, \ldots, A_{u}$ are fixed)

$$
\mathcal{M}=\max _{\underline{Z} \in \mathcal{D}} \frac{A_{1} A_{2} \cdots A_{u}}{f(\underline{Z})}
$$

where $\mathcal{D}$ is a subset of $\mathbb{N}^{r+1+u}, \underline{Z}=\left(Z_{1}, Z_{2}, \ldots, Z_{r+1+u}\right)$,

$$
f(\underline{Z})=Z_{1} Z_{2} \cdots Z_{r+1+u}
$$

and the set $\mathcal{D}$ is defined by

$$
\begin{gathered}
Z_{i} \geqslant p_{i}, \quad 1 \leqslant i \leqslant r+1, \\
Z_{i}<Z_{r+1}, \quad 1 \leqslant i \leqslant r, \\
Z_{r+1}<Z_{r+1+i} \leqslant A_{i}, \quad 1 \leqslant i \leqslant u,
\end{gathered}
$$

and

$$
U(\underline{Z})-R(\underline{Z})-Z_{r+1}+\sigma_{j+r+1}-\sigma_{r}=\nu
$$

with

$$
U(\underline{Z})=\sum_{i=1}^{u}\left(A_{i}-Z_{r+1+i}\right), \quad R(\underline{Z})=\sum_{i=1}^{r}\left(Z_{i}-p_{i}\right) .
$$

Note that, from (4.5) and (4.8), $\underline{B} \in \mathcal{D}$ so that (4.4) implies

$$
\frac{h_{j}(n)}{N_{j+r+1}}=\frac{A_{1} A_{2} \cdots A_{u}}{f(\underline{B})} \leqslant \mathcal{M}=\max _{\underline{Z} \in \mathcal{D}} \frac{A_{1} A_{2} \cdots A_{u}}{f(\underline{Z})} .
$$

If $\underline{Z} \in \mathcal{D}$, from (4.10)-(4.12), it follows that

$$
2=p_{1} \leqslant Z_{i} \leqslant A_{u}, \quad 1 \leqslant i \leqslant r+1+u,
$$

so that $f(\underline{Z})$ does not vanish on $\mathcal{D}$ and $\mathcal{D}$ is finite. Therefore, the maximum $\mathcal{M}$ defined by (4.9) is finite; let $\underline{C}$ be a point in $\mathcal{D}$ where the maximum $\mathcal{M}$ is attained. We shall prove that

$$
U(\underline{C})=R(\underline{C})=0 .
$$

For that, first we claim that one of the two numbers $U(\underline{C}), R(\underline{C})$ vanishes. Indeed, assume that $U(\underline{C}) \neq 0$ and $R(\underline{C}) \neq 0$. From (4.10), we have $R(\underline{C})>0$; thus there exists $i_{0}, 1 \leqslant i_{0} \leqslant r$, such that

$$
C_{i_{0}} \geqslant p_{i_{0}}+1>p_{i_{0}} .
$$

Similarly, from (4.12), we have $U(\underline{C})>0$, and there exists $i_{1}, 1 \leqslant i_{1} \leqslant u$, such that

$$
A_{i_{1}}>A_{i_{1}}-1 \geqslant C_{r+1+i_{1}} .
$$

Let us define $\underline{C}^{\prime} \in \mathbb{N}^{r+1+u}$ by

$$
C_{i_{0}}^{\prime}=C_{i_{0}}-1, \quad C_{r+1+i_{1}}^{\prime}=C_{r+1+i_{1}}+1, \quad C_{i}^{\prime}=C_{i}, \quad i \neq i_{0}, r+1+i_{1} .
$$

To prove that $\underline{C}^{\prime} \in \mathcal{D}$, we have to check that $C_{i_{0}}^{\prime} \geqslant p_{i_{0}}$ (which follows from (4.17)), that $C_{i_{0}}^{\prime}<C_{r+1}$ (which follows from $C_{i_{0}}^{\prime}=C_{i_{0}}-1<C_{r+1}-1$ ), that $C_{r+1}<C_{r+1+i_{1}}^{\prime}$ (since $C_{r+1}<C_{r+1+i_{1}}$ and $\left.C_{r+1+i_{1}}=C_{r+1+i_{1}}^{\prime}-1\right)$, that $C_{r+1+i_{1}}^{\prime} \leqslant A_{i_{1}}$ (which follows from (4.18)) and that $U\left(\underline{C^{\prime}}\right)-R\left(\underline{C}^{\prime}\right)=$ $U(\underline{C})-R(\underline{C})$ (which follows from $U\left(\underline{C}^{\prime}\right)=U(\underline{C})-1$ and $R\left(\underline{C}^{\prime}\right)=R(\underline{C})-1$ ). Further, we have

$$
\frac{f\left(\underline{C^{\prime}}\right)}{f(\underline{C})}=\frac{C_{i_{0}}^{\prime} C_{r+1+i_{1}}^{\prime}}{C_{i_{0}} C_{r+1+i_{1}}}=\frac{\left(C_{i_{0}}-1\right)\left(C_{r+1+i_{1}}+1\right)}{C_{i_{0}} C_{r+1+i_{1}}}=1-\frac{\left(C_{r+1+i_{1}}-C_{i_{0}}+1\right)}{C_{i_{0}} C_{r+1+i_{1}}}<1
$$


since, from the definition of $\mathcal{D}$ (cf. (4.11) and (4.12)), $C_{i_{0}}<C_{r+1}<C_{r+1+i_{1}}$ holds. But (4.19) contradicts the fact that the maximum in (4.9) is attained at $\underline{C}$.

Let us show now that it is impossible to have simultaneously $U(\underline{C})>0$ and $R(\underline{C})=0$; indeed, let us assume that $U(\underline{C}) \geqslant 1$ and $R(\underline{C})=0$ (which implies $r=0$ or $C_{i}=p_{i}$ for $1 \leqslant i \leqslant r$ ). We define $i_{1}$ as in (4.18). Since $\underline{C} \in \mathcal{D}$, we get from (4.13)

$$
C_{r+1}=\sigma_{j+r+1}-\sigma_{r}-\nu+U(\underline{C})=\left(\sigma_{j+r+1}-\sigma_{r+1}-\nu\right)+p_{r+1}+U(\underline{C})
$$

which, by $(4.6)$ and $U(\underline{C}) \geqslant 1$, yield

$$
C_{r+1}>p_{r+1}+U(\underline{C}) \geqslant p_{r+1}+1 .
$$

We define $\underline{C}^{\prime} \in \mathbb{N}^{r+1+u}$ by

$$
C_{r+1}^{\prime}=C_{r+1}-1, \quad C_{r+1+i_{1}}^{\prime}=C_{r+1+i_{1}}+1, \quad C_{i}^{\prime}=C_{i}, \quad i \neq r+1, r+1+i_{1} .
$$

To prove that $\underline{C}^{\prime} \in \mathcal{D}$, we have to check that $C_{i}^{\prime} \geqslant p_{i}$ for $1 \leqslant i \leqslant r+1$ (which follows from $C_{i}^{\prime}=C_{i}=p_{i}$ if $i \leqslant r$ and from (4.20) if $i=r+1$ ), that $C_{i}^{\prime}<C_{r+1}^{\prime}$ for $1 \leqslant i \leqslant r$ (which follows from $C_{i}^{\prime}=C_{i}=p_{i} \leqslant p_{r}$ and from $C_{r+1}^{\prime}=C_{r+1}-1 \geqslant p_{r+1}$, via(4.20)), that $C_{r+1}^{\prime}<C_{r+1+i}^{\prime}$ for $1 \leqslant i \leqslant u$ (which follows from $C_{r+1}^{\prime}<C_{r+1}$ and $C_{r+1+i}^{\prime} \geqslant C_{r+1+i}$ ), that $C_{r+1+i_{1}}^{\prime} \leqslant A_{i_{1}}$ (which follows from (4.18)) and that $\left.U\left(\underline{C}^{\prime}\right)-C_{r+1}^{\prime}=U(\underline{C})-C_{r+1}\right)$ (which is easy). As in (4.19), we have $f\left(\underline{C}^{\prime}\right)<f(\underline{C})$, contradicting the fact that the maximum in $(4.9)$ is attained at $\underline{C}$.

To prove (4.16), it remains to show that we cannot have $R(\underline{C})>0$ and $U(\underline{C})=0$. Let us suppose that $R(\underline{C}) \geqslant 1$ and $U(\underline{C})=0$, which implies either $u=0$ or, for $1 \leqslant i \leqslant u$,

$$
C_{r+1+i}=A_{i} \geqslant p_{j+r+2} \geqslant p_{j+r+1}+2,
$$

with the help of (4.5). From (4.13) and (4.7), this time we get

$$
\begin{aligned}
C_{r+1} & =\sigma_{j+r+1}-\sigma_{r}-\nu-R(\underline{C}) \\
& \leqslant \sigma_{j+r+1}-\sigma_{r}-\left(\sigma_{j+r}-\sigma_{r}\right)-R(\underline{C})=p_{j+r+1}-R(\underline{C}) \leqslant p_{j+r+1}-1 .
\end{aligned}
$$

Here we choose $i_{0}$ as in (4.17) and set

$$
C_{i_{0}}^{\prime}=C_{i_{0}}-1, \quad C_{r+1}^{\prime}=C_{r+1}+1, \quad C_{i}^{\prime}=C_{i}, \quad i \neq i_{0}, r+1 .
$$

To prove that $\underline{C}^{\prime} \in \mathcal{D}$, we have to check that $C_{i_{0}}^{\prime} \geqslant p_{i_{0}}$ (which follows from (4.17)), that $C_{r+1}^{\prime} \geqslant p_{r+1}$ (which follows from $C_{r+1}^{\prime}=C_{r+1}+1$ and $C_{r+1} \geqslant p_{r+1}$ ), that, for $1 \leqslant i \leqslant r, C_{i}^{\prime}<C_{r+1}^{\prime}$ (which follows from $C_{i}^{\prime} \leqslant C_{i}$ and $C_{r+1}^{\prime}>C_{r+1}$ ), that, for $1 \leqslant i \leqslant u, C_{r+1}^{\prime}<C_{r+1+i_{1}}=A_{i_{1}}$ (which follows from (4.21) and (4.22)) and that $R\left(\underline{C}^{\prime}\right)+C_{r+1}^{\prime}=R(\underline{C})+C_{r+1}$ (which is easy). As precedingly in (4.19), we observe that $f\left(\underline{C}^{\prime}\right)<f(\underline{C})$, contradicting the fact that the minimum is attained at $\underline{C}$.

In conclusion, we have proved (4.16) so that $C_{i}=p_{i}$ for $1 \leqslant i \leqslant r$ and $C_{r+1+i}=A_{i}$ for $1 \leqslant i \leqslant u$. Moreover, (4.6) yields $\nu \leqslant n$, and, from (4.13) and (4.2), we get

$$
C_{r+1}=\sigma_{j+r+1}-\sigma_{r}-\nu \geqslant \sigma_{j+r+1}-\sigma_{r}-n=p_{j+r+1}-n^{\prime} .
$$

Therefore, the maximum $\mathcal{M}$ in (4.9) satisfies

$$
\mathcal{M}=\frac{A_{1} A_{2} \cdots A_{u}}{p_{1} p_{2} \cdots p_{r} C_{r+1} A_{1} A_{2} \cdots A_{u}} \leqslant \frac{1}{p_{1} p_{2} \cdots p_{r}\left(p_{j+r+1}-n^{\prime}\right)}
$$

which, via (4.15), proves (4.3). 
Proposition 4.2. With the notation of Proposition 4.1, we have

$$
h_{j}(n) \geqslant \frac{N_{j+r+1}}{N_{r}\left(p_{j+r+1}-n^{\prime}\right)^{\star}}=\frac{N_{j+r+1}}{q N_{r}}
$$

where $q=\left(p_{j+r+1}-n^{\prime}\right)^{\star}$ is the smallest prime satisfying $q \geqslant p_{j+r+1}-n^{\prime}$.

Proof. From (4.2), we have $p_{r+1}<p_{j+r+1}-n^{\prime} \leqslant p_{j+r+1}$ which implies $p_{r+1} \leqslant q \leqslant p_{j+r+1}$ so that $M=N_{j+r+1} /\left(q N_{r}\right)$ is an integer with exactly $j$ prime factors. Further, by (4.2), we have

$$
\ell(M)=\sigma_{j+r+1}-\sigma_{r}-q \leqslant \sigma_{j+r+1}-\sigma_{r}-\left(p_{j+r+1}-n^{\prime}\right)=n
$$

and, by $(1.9), h_{j}(n) \geqslant M$ holds.

COROllary 4.1. We keep the notation of Proposition 4.1 ; if $q=p_{j+r+1}-n^{\prime}$ is prime then

$$
h_{j}(n)=h_{j}\left(\sigma_{j+r+1}-\sigma_{r}-q\right)=\frac{N_{j+r+1}}{q N_{r}} .
$$

Proof. Corollary 4.1 follows from Propositions 4.1 and 4.2.

\section{A parity phenomenon}

Proposition 5.1. Let $k \geqslant 2$ be an integer and $a$ be an even number satisfying $4 \leqslant a<p_{k+1}$ and $h_{k}$ defined by (1.9). We have

$$
h_{k}\left(\sigma_{k+1}-a\right)=h_{k}\left(\sigma_{k+1}-a-1\right) .
$$

Proof. Since $n \mapsto h_{k}(n)$ in non-decreasing, we have

$$
h_{k}\left(\sigma_{k+1}-a\right) \geqslant h_{k}\left(\sigma_{k+1}-a-1\right) .
$$

Let us set $n=\sigma_{k+1}-a$ and note that $n$ satisfies $\sigma_{k}<n<\sigma_{k+1}$ so that, from (1.8), $k=$ $k(n)=k(n-1)$. Let $M$ be a positive squarefree integer such that $\ell(M) \leqslant n$ and $\omega(M)=k$. Such a number $M$ is even; if not, we would have $\ell(M) \geqslant 3+5+\cdots+p_{k+1}=\sigma_{k+1}-2$ in contradiction with $\ell(M) \leqslant n=\sigma_{k+1}-a \leqslant \sigma_{k+1}-4$. Therefore, $\ell(M)$ is the sum of 2 and $k-1$ odd numbers, so that $\ell(M) \equiv \sigma_{k} \equiv \sigma_{k+1}+1 \equiv \sigma_{k+1}-a-1(\bmod 2)$. So, $\ell(M)$ cannot be equal to $\sigma_{k+1}-a$ and $\ell(M) \leqslant \sigma_{k+1}-a-1$ holds. Thus, from (1.9), we get $h_{k}\left(\sigma_{k+1}-a\right) \leqslant h_{k}\left(\sigma_{k+1}-a-1\right)$, which, with (5.2), proves (5.1).

Proposition 5.2. Let $k$ be an integer, $k \geqslant 2$, and $q$ a prime number satisfying $3 \leqslant q \leqslant p_{k}$. By setting $m=\sigma_{k+1}-q-1$, we have

$$
h_{k-1}(m)=h_{k-1}(m-1)=h_{k-1}\left(\sigma_{k+1}-q-2\right)=\frac{N_{k+1}}{2 q} .
$$

Proof. From Table 1, we have $h_{1}(6)=h_{1}(5)=5, h_{2}(11)=h_{2}(10)=21$ and $h_{2}(13)=h_{2}(12)=$ 35 so that the proposition is true for $k=2, q=3$ and for $k=3$ and $q=3$ or 5 . So, from now on, we assume $k \geqslant 4$. Corollary 4.1 with $j=k-1, r=1$ implies $h_{k-1}(m-1)=N_{k+1} /(2 q)$ and, since $n \mapsto h_{k-1}(n)$ is non-decreasing, it follows that

$$
h_{k-1}(m) \geqslant h_{k-1}(m-1)=\frac{N_{k+1}}{2 q} .
$$

Let $M$ be a positive squarefree integer satisfying $\ell(M) \leqslant m$ and $\omega(M)=k-1$. In view of (5.4) and (1.9), to prove that $h_{k-1}(m)=N_{k+1} /(2 q)$, it suffices to show that

$$
M \leqslant \frac{N_{k+1}}{2 q} .
$$


If $M$ is odd, $\ell(M)$ is the sum of $k-1$ odd numbers, which implies

$$
\ell(M) \equiv \sigma_{k} \equiv \sigma_{k+1}-q=m+1(\bmod 2)
$$

So, $\ell(M)$ cannot be equal to $m$; since, by $(1.9), \ell(M) \leqslant m$ holds, we should have $\ell(M) \leqslant m-1$; therefore, from (1.9), we get

$$
M \leqslant h_{k-1}(m-1)=\frac{N_{k+1}}{2 q} .
$$

If $M$ is even, we have $\omega(M / 2)=k-2$ and $\ell(M / 2) \leqslant m-2$, so that

$$
M \leqslant 2 h_{k-2}(m-2) \text {. }
$$

If $q \geqslant 11$, since we have assumed $k \geqslant 4$, i.e. $p_{k+1} \geqslant 11$, we have

$$
\sigma_{k}-5 \leqslant m-2=\sigma_{k+1}-q-3 \leqslant \sigma_{k+1}-14<\sigma_{k+1}-10 .
$$

By Proposition 4.1 with $j=k-2, r=2, n=m-2, n^{\prime}=n-\left(\sigma_{k}-\sigma_{2}\right)=p_{k+1}-q+2$, we get

$$
h_{k-2}(m-2) \leqslant \frac{N_{k+1}}{6(q-2)}
$$

which, by (5.7), gives

$$
M \leqslant \frac{N_{k+1}}{3(q-2)}=\frac{N_{k+1}}{3 q} \frac{q}{q-2} \leqslant \frac{N_{k+1}}{3 q} \frac{11}{9}<\frac{N_{k+1}}{2 q} .
$$

If $q \in\{3,5,7\}$, since $k \geqslant 4$ and $p_{k+2} \geqslant p_{6}=13$, we have

$$
\sigma_{k+1}-10 \leqslant m-2=\sigma_{k+1}-q-3 \leqslant \sigma_{k+1}-6<\sigma_{k+2}-17 .
$$

and Proposition 4.1 with $j=k-2, r=3, n=m-2$ and $n^{\prime}=n-\left(\sigma_{k+1}-10\right)=7-q$ yields

$$
h_{k-2}(m-2) \leqslant \frac{N_{k+2}}{30\left(p_{k+2}+q-7\right)} .
$$

Using $q \leqslant 7, k \geqslant 4$ and $p_{k+2} \geqslant p_{6}=13$ gives

$$
\frac{N_{k+2}}{30\left(p_{k+2}+q-7\right)}=\frac{N_{k+1}}{30} \frac{p_{k+2}}{p_{k+2}+q-7} \leqslant \frac{N_{k+1}}{30} \frac{13}{q+6}=\frac{13 N_{k+1}}{30 q} \frac{q}{q+6} \leqslant \frac{13 N_{k+1}}{30 q} \frac{7}{13}<\frac{N_{k+1}}{4 q}
$$

which, together with (5.7) and (5.9), proves

$$
M<\frac{N_{k+1}}{2 q} .
$$

Inequalities (5.6), (5.8) and (5.10) prove that (5.5) holds, which, with (5.4), completes the proof of $(5.3)$.

Proposition 5.3. Let $k$ be a positive integer and $m=\sigma_{k+1}-1$; we have

$$
h_{k}(m)=h_{k}(m-1)=h_{k}\left(\sigma_{k+1}-2\right)=\frac{N_{k+1}}{2} .
$$


Proof. It is the same proof than for Proposition 5.2. By Proposition 3.2 with $j=k$ and $r=1$, we have

$$
h_{k}(m) \geqslant h_{k}(m-1)=\frac{N_{k+1}}{2} .
$$

Further, let $M$ be a positive integer satisfying $\ell(M) \leqslant m$ and $\omega(M)=k$. If $M$ is odd, by the parity phenomenon, we have $\ell(M) \equiv m-1(\bmod 2)$ so that $\ell(M) \leqslant m-1$ and $M \leqslant h_{k}(m-1)=N_{k+1} / 2$. If $M$ is even, we have $M \leqslant 2 h_{k-1}\left(\sigma_{k+1}-3\right)$ and, if $k \geqslant 2$, i.e. $p_{k+2} \geqslant 7$, Proposition 4.1 with $j=k-1, r=2, n=\sigma_{k+1}-3, n^{\prime}=2$, yields

$$
M \leqslant 2 \frac{N_{k+2}}{6\left(p_{k+2}-2\right)}=\frac{N_{k+1}}{3} \frac{p_{k+2}}{p_{k+2}-2} \leqslant \frac{7 N_{k+1}}{15}<\frac{N_{k+1}}{2} .
$$

If $k=1$, it is easy to check that (5.11) still holds.

\section{The increasingness of $h_{j}(n)$ on $j$}

THEOREM 6.1. Let $n \geqslant 2$ be an integer and $k=k(n)$ be defined by (1.8); for $j$ satisfying $1 \leqslant j \leqslant k$, we have

$$
h_{j-1}(n) \leqslant \frac{5}{6} h_{j}(n)
$$

and (6.1) is an equality if and only if $j=k(n) \geqslant 2$ and $n=\sigma_{j+1}-4$ or $n=\sigma_{j+1}-5$.

Proof. If $j=1$, it follows from (1.10) and (1.11) that $h_{0}(n)=1, h_{1}(n)={ }^{\star}(n) \geqslant 2$ and $h_{0}(n) / h_{1}(n) \leqslant 1 / 2<5 / 6$, which proves (6.1). So, from now on, we assume $j \geqslant 2$. by

The sequence $\left(\sigma_{j+r}-\sigma_{r}\right)_{r \geqslant 0}$ is increasing and goes to infinity. So, we may define $r_{j} \geqslant 0$ and $n_{j}^{\prime}$

$$
\sigma_{j+r_{j}}-\sigma_{r_{j}} \leqslant n<\sigma_{j+r_{j}+1}-\sigma_{r_{j}+1}
$$

and

$$
n_{j}^{\prime}=n-\left(\sigma_{j+r_{j}}-\sigma_{r_{j}}\right) .
$$

We shall consider four cases: $r_{j} \leqslant j-4, r_{j} \geqslant j+3, j-3 \leqslant r_{j} \leqslant j+2$ and $j \geqslant 25, j-3 \leqslant r_{j} \leqslant$ $j+2$ and $j \leqslant 24$.

First case: $r_{j} \leqslant j-4$. From (2.5) and our hypothesis $j \geqslant r_{j}+4$, we deduce

$$
p_{r_{j}+1}+p_{r_{j}+2} \leqslant p_{2 r_{j}+3}<p_{j+r_{j}}<p_{j+r_{j}+1}
$$

and

$$
p_{r_{j}+2}+p_{r_{j}+3} \leqslant p_{2 r_{j}+5}<p_{j+r_{j}+2}
$$

From (6.3) and (6.2), we get

$$
0 \leqslant n_{j}^{\prime}=n-\left(\sigma_{j+r_{j}}-\sigma_{r_{j}}\right)<p_{j+r_{j}+1}-p_{r_{j}+1}
$$

and applying Proposition 4.2 yield

$$
h_{j}(n) \geqslant \frac{N_{j+r_{j}+1}}{q N_{r_{j}}}=\frac{N_{j+r_{j}+1}}{N_{r_{j}}\left(p_{j+r_{j}+1}-n_{j}^{\prime}\right)^{\star}} .
$$

In view of bounding $h_{j-1}(n)$, we have to determine $r_{j-1}$ such that

$$
\sigma_{j-1+r_{j-1}}-\sigma_{r_{j-1}} \leqslant n<\sigma_{j+r_{j-1}}-\sigma_{r_{j-1}+1} .
$$

We shall distinguish two sub cases. 
Subcase one: $r_{j-1}=r_{j}+1$. Let us assume that

$$
\sigma_{j+r_{j}}-\sigma_{r_{j}} \leqslant n<\sigma_{j+r_{j}+1}-\sigma_{r_{j}+2},
$$

i.e. from (6.3),

$$
0 \leqslant n_{j}^{\prime}=n-\left(\sigma_{j+r_{j}}-\sigma_{r_{j}}\right)<p_{j+r_{j}+1}-p_{r_{j}+1}-p_{r_{j}+2} .
$$

Note that, from (6.4), the right hand side of (6.10) is positive. Then, we have $r_{j-1}=r_{j}+1$ since, from (6.9),

$$
\begin{aligned}
\sigma_{(j-1)+\left(r_{j}+1\right)}-\sigma_{r_{j}+1} & =\sigma_{j+r_{j}}-\sigma_{r_{j}+1}<\sigma_{j+r_{j}}-\sigma_{r_{j}} \leqslant n \\
& <\sigma_{j+r_{j}+1}-\sigma_{r_{j}+2}=\sigma_{(j-1)+\left(r_{j}+1\right)+1}-\sigma_{\left(r_{j}+1\right)+1}
\end{aligned}
$$

holds. Via (6.3), this implies that

$$
n_{j-1}^{\prime} \stackrel{\text { def }}{=} n-\left(\sigma_{j-1+r_{j-1}}-\sigma_{r_{j-1}}\right)=n-\sigma_{j+r_{j}}+\sigma_{r_{j}+1}=n_{j}^{\prime}+p_{r_{j}+1} .
$$

Applying Proposition 4.1 and noting that $j-1+r_{j-1}=j+r_{j}$ yield

$$
h_{j-1}(n) \leqslant \frac{N_{j-1+r_{j-1}+1}}{N_{r_{j-1}}\left(p_{j-1+r_{j-1}+1}-n_{j-1}^{\prime}\right)}=\frac{N_{j+r_{j}+1}}{N_{r_{j}+1}\left(p_{j+r_{j}+1}-n_{j}^{\prime}-p_{r_{j}+1}\right)} .
$$

By using (6.7), we get

$$
\frac{h_{j-1}(n)}{h_{j}(n)} \leqslant \frac{\left(p_{j+r_{j}+1}-n_{j}^{\prime}\right)^{\star}}{p_{r_{j}+1}\left(p_{j+r_{j}+1}-n_{j}^{\prime}-p_{r_{j}+1}\right)} .
$$

From (6.6), we have $p_{j+r_{j}+1}-n_{j}^{\prime}>p_{r_{j}+1} \geqslant p_{1}=2$, so that we may apply Lemma 2.1 which, with the help of (6.11) and (6.10), yields

$$
\frac{h_{j-1}(n)}{h_{j}(n)} \leqslant \frac{11}{8 p_{r_{j}+1}}\left(1+\frac{p_{r_{j}+1}}{p_{j+r_{j}+1}-n_{j}^{\prime}-p_{r_{j}+1}}\right)<\frac{11}{8}\left(\frac{1}{p_{r_{j}+1}}+\frac{1}{p_{r_{j}+2}}\right) .
$$

If $r_{j} \geqslant 1$,

$$
\frac{11}{8}\left(\frac{1}{p_{r_{j}+1}}+\frac{1}{p_{r_{j}+2}}\right) \leqslant \frac{11}{8}\left(\frac{1}{3}+\frac{1}{5}\right)<\frac{5}{6}
$$

which proves (6.1).

It remains to consider the case $r_{j}=0$, which, from (6.9) and (1.8), implies $\sigma_{j} \leqslant n<\sigma_{j+1}$ and $k(n)=j$.

Then (6.10) becomes $0 \leqslant n_{j}^{\prime}=n-\sigma_{j}<p_{j+1}-5$ and, by setting $a=p_{j+1}-n_{j}^{\prime}$, we get

$$
5<a=p_{j+1}-n_{j}^{\prime}=p_{j+1}+\sigma_{j}-n=\sigma_{j+1}-n<p_{j+1}
$$

while (6.11) yields

$$
\frac{h_{j-1}(n)}{h_{j}(n)} \leqslant \frac{(a)^{\star}}{2(a-2)} .
$$

By Lemma $2.1,(a)^{\star} \leqslant(11 / 8) a$ holds, and, for $a \geqslant 12$,

$$
\frac{11}{16} \frac{a}{a-2} \leqslant \frac{11}{16} \frac{12}{10}<\frac{5}{6}
$$

which, via (6.13), proves (6.1).

Since, from (6.12), $a>5$, it remains to study the cases $6 \leqslant a \leqslant 11$. If $a=7,9,10,11$, it is easy to check that

$$
\frac{(a)^{\star}}{2(a-2)}<\frac{5}{6}
$$


If $a=6$ or $a=8$, by Proposition 5.1, (6.12) and (4.23) we have

$$
h_{j}(n)=h_{j}\left(\sigma_{j+1}-a\right)=h_{j}\left(\sigma_{j+1}-a-1\right)=h_{j}(n-1) \geqslant \frac{N_{j+1}}{(a+1)^{\star}}
$$

while, by Proposition 5.2, since $a-1$ is prime, we get

$$
h_{j-1}(n)=h_{j-1}(n-1)=\frac{N_{j+1}}{2(a-1)}
$$

yielding

$$
\frac{h_{j-1}(n)}{h_{j}(n)}=\frac{h_{j-1}(n-1)}{h_{j}(n-1)} \leqslant \frac{(a+1)^{\star}}{2(a-1)}= \begin{cases}\frac{7}{10} & \text { if } a=6, \\ \frac{11}{14} & \text { if } a=8,\end{cases}
$$

and, in both cases,

$$
\frac{h_{j-1}(n)}{h_{j}(n)}<\frac{5}{6}
$$

holds, which proves (6.1).

Subcase two: $r_{j-1}=r_{j}+2$. Now, we assume that (6.2) holds but not (6.9); thus we have

$$
\sigma_{j+r_{j}+1}-\sigma_{r_{j}+2} \leqslant n<\sigma_{j+r_{j}+1}-\sigma_{r_{j}+1}
$$

and, from (6.5),

$$
p_{j+r_{j}+1}-p_{r_{j}+1}-p_{r_{j}+2} \leqslant n_{j}^{\prime}<p_{j+r_{j}+1}-p_{r_{j}+1} .
$$

Here, we get $r_{j-1}=r_{j}+2$, since we have

$$
\sigma_{j-1+r_{j}+2}-\sigma_{r_{j}+2} \leqslant n<\sigma_{j+r_{j}+1}-\sigma_{r_{j}+1}<\sigma_{j+r_{j}+2}-\sigma_{r_{j}+3}
$$

by observing that, from (6.5),

$$
\sigma_{j+r_{j}+2}-\sigma_{r_{j}+3}-\left(\sigma_{j+r_{j}+1}-\sigma_{r_{j}+1}\right)=p_{j+r_{j}+2}-p_{r_{j}+2}-p_{r_{j}+3}>0
$$

holds. Now, we have

$$
\begin{aligned}
0 \leqslant n_{j-1}^{\prime} & =n-\left(\sigma_{j-1+r_{j-1}}-\sigma_{r_{j-1}}\right)=n-\sigma_{j+r_{j}+1}+\sigma_{r_{j}+2} \\
& =n_{j}^{\prime}+\sigma_{j+r_{j}}-\sigma_{r_{j}}-\sigma_{j+r_{j}+1}+\sigma_{r_{j}+2} \\
& =n_{j}^{\prime}-p_{j+r_{j}+1}+p_{r_{j}+1}+p_{r_{j}+2} \\
& <p_{r_{j}+2}
\end{aligned}
$$

and applying Proposition 4.1 gives

$$
h_{j-1}(n) \leqslant \frac{N_{j-1+r_{j-1}+1}}{N_{r_{j-1}}\left(p_{j-1+r_{j-1}+1}-n_{j-1}^{\prime}\right)}=\frac{N_{j+r_{j}+2}}{N_{r_{j}+2}\left(p_{j+r_{j}+2}-n_{j-1}^{\prime}\right)}
$$

while Proposition 4.2 yields

$$
h_{j}(n) \geqslant \frac{N_{j+r_{j}+1}}{N_{r_{j}}\left(p_{j+r_{j}+1}-n_{j}^{\prime}\right)^{\star}} .
$$

We set $a=p_{j+r_{j}+1}-n_{j}^{\prime}$ and $\Delta=p_{j+r_{j}+2}-p_{r_{j}+1}-p_{r_{j}+2}$ so that (6.16) allows to write $p_{j+r_{j}+2}-$ $n_{j-1}^{\prime}=\Delta+a$, and we have

$$
\frac{h_{j-1}(n)}{h_{j}(n)} \leqslant \frac{p_{j+r_{j}+2}}{p_{r_{j}+1} p_{r_{j}+2}} \frac{(a)^{\star}}{\Delta+a} .
$$


Formula (6.16) can be rewritten as

$$
2=p_{1} \leqslant p_{r_{j}+1}<a=p_{j+r_{j}+1}-n_{j}^{\prime} \leqslant p_{r_{j}+1}+p_{r_{j}+2} .
$$

Lemma 2.1 implies $(a)^{\star} \leqslant 11 a / 8$ and, by (6.4), $\Delta>0$ holds, so that the homographic function $t \mapsto t /(\Delta+t)$ is increasing. From (6.18), we thus have

$$
\frac{a}{\Delta+a} \leqslant \frac{p_{r_{j}+1}+p_{r_{j}+2}}{\Delta+p_{r_{j}+1}+p_{r_{j}+2}}=\frac{p_{r_{j}+1}+p_{r_{j}+2}}{p_{j+r_{j}+2}} .
$$

Therefore, we get

$$
\frac{h_{j-1}(n)}{h_{j}(n)} \leqslant \frac{11}{8} \frac{p_{r_{j}+1}+p_{r_{j}+2}}{p_{r_{j}+1} p_{r_{j}+2}}=\frac{11}{8}\left(\frac{1}{p_{r_{j}+1}}+\frac{1}{p_{r_{j}+2}}\right)
$$

which is smaller than $5 / 6$ if $r_{j} \geqslant 1$.

It remains to consider the case $r_{j}=0, r_{j-1}=2$. Formula (6.17) becomes

$$
\frac{h_{j-1}(n)}{h_{j}(n)} \leqslant \frac{p_{j+2}}{6} \frac{(a)^{\star}}{p_{j+2}-5+a}
$$

while (6.18) via (6.3) becomes

$$
2<a=p_{j+1}-n_{j}^{\prime}=\sigma_{j+1}-n \leqslant 5 .
$$

Since $j \geqslant 2$ holds, note that (6.20) implies $\sigma_{j} \leqslant \sigma_{j+1}-5 \leqslant n<\sigma_{j+1}-2$, which shows from (1.8) that $k(n)=j$.

If $a=5$, since $n=\sigma_{j+1}-a=\sigma_{j+1}-5$, by Corollary 4.1 with $r=r_{j}=0$ and $q=5$, we get $h_{j}(n)=N_{j+1} / 5$, while Proposition 3.2 gives

$$
h_{j-1}\left(\sigma_{j+1}-5\right)=h_{j-1}\left(\sigma_{j+1}-\sigma_{2}\right)=\frac{N_{j+1}}{N_{2}}=\frac{N_{j+1}}{6} .
$$

Therefore, $h_{j-1}\left(\sigma_{j+1}-5\right) /\left(h_{j}\left(\sigma_{j+1}-5\right)\right)=5 / 6$.

If $a=4$, by Proposition 5.1, we get $h_{j}\left(\sigma_{j+1}-4\right)=h_{j}\left(\sigma_{j+1}-5\right)$ and, by Proposition 5.2, $h_{j-1}\left(\sigma_{j+1}-4\right)=h_{j-1}\left(\sigma_{j+1}-5\right)$ so that

$$
\frac{h_{j-1}\left(\sigma_{j+1}-4\right)}{h_{j}\left(\sigma_{j+1}-4\right)}=\frac{h_{j-1}\left(\sigma_{j+1}-5\right)}{h_{j}\left(\sigma_{j+1}-5\right)}=\frac{5}{6} .
$$

If $a=3$, Formula (6.19) becomes

$$
\frac{h_{j-1}(n)}{h_{j}(n)} \leqslant \frac{p_{j+2}}{2\left(p_{j+2}-2\right)} \leqslant \frac{7}{10}<\frac{5}{6}
$$

since $p_{j+2} \geqslant p_{4}=7$.

Second case: $r_{j} \geqslant j+3$. From (6.2), we deduce $n \geqslant \sigma_{j+r_{j}}-\sigma_{r_{j}+1}=\sigma_{(j-1)+\left(r_{j}+1\right)}-\sigma_{\left(r_{j}+1\right)}$ and Proposition 3.2, (3.3), implies

$$
\ell\left(h_{j-1}(n)\right) \geqslant \sigma_{j+r_{j}}-\sigma_{r_{j}+1}
$$

Let us now show that

$$
q \stackrel{\text { def }}{=} P^{+}\left(h_{j-1}(n)\right) \geqslant p_{j+r_{j}} .
$$

Indeed, if $q \leqslant p_{j+r_{j}-1}$ holds, since $h_{j-1}(n)$ has $j-1$ prime factors, we should have

$$
\ell\left(h_{j-1}(n)\right) \leqslant p_{j+r_{j}-1}+p_{j+r_{j}-2}+\cdots+p_{r_{j}+1}<p_{j+r_{j}}+p_{j+r_{j}-1}+\cdots+p_{r_{j}+2}=\sigma_{j+r_{j}}-\sigma_{r_{j}+1}
$$

which would contradict (6.21). 
Further, among the $j+1$ primes $p_{2}=3, p_{3}, \ldots, p_{j+2}$, there are certainly two primes $p$ and $p^{\prime}$ not dividing $h_{j-1}(n)$ and satisfying $3 \leqslant p<p^{\prime} \leqslant p_{j+2}$. By Lemma 2.4, (2.5), and (6.22), we get

$$
p+p^{\prime} \leqslant p_{j+1}+p_{j+2} \leqslant p_{2 j+3} \leqslant p_{j+r_{j}} \leqslant q=P^{+}\left(h_{j-1}(n)\right)
$$

and, applying Proposition 3.4 proves

$$
\frac{h_{j-1}(n)}{h_{j}(n)}<\frac{5}{6} .
$$

Third case: $j-3 \leqslant r_{j} \leqslant j+2$ and $j \geqslant 25$. The proof is the same than for the second case; only, in (6.23), instead of (2.5), we use (2.6) with $b=7, i=j+2 \geqslant 27$ :

$$
p+p^{\prime} \leqslant p_{j+1}+p_{j+2}<p_{2 j-3} \leqslant p_{j+r_{j}} \leqslant q=P^{+}\left(h_{j-1}(n)\right) .
$$

Fourth case: $j-3 \leqslant r_{j} \leqslant j+2$ and $j \leqslant 24$. Here, we have $r_{j} \leqslant j+2 \leqslant 26$ and, from (6.2), we get

$$
n<\sigma_{j+r_{j}+1} \leqslant \sigma_{51}=5350 .
$$

So, for $k \leqslant 50, \sigma_{k} \leqslant n<\sigma_{k+1}$ and $1 \leqslant j \leqslant k$, we have computed $h_{j}(n)$ with the algorithm described in Section 1.4 and we have checked that, for $j \geqslant 2$,

$$
\frac{h_{j-1}(n)}{h_{j}(n)} \leqslant \frac{5}{6}
$$

always holds, with equality if and only if $j=k(n)$ and $n=\sigma_{j+1}-4$ or $n=\sigma_{j+1}-5$.

Corollary 6.1. For all non-negative integer $n \geqslant 2$, we have

$$
h(n)=h_{k}(n)
$$

where $k=k(n)$ is defined by (1.8).

Proof. From (1.1) and (1.9) we have

$$
h(n)=\max _{0 \leqslant j \leqslant k(n)} h_{j}(n)
$$

and Theorem 6.1 yields $h_{0}(n)<h_{1}(n)<\cdots<h_{k(n)}(n)$.

\section{Computation of $\pi_{f}(x)$}

Let $f$ be an arithmetic function, i.e. a function defined on positive integers. The simplest way to compute $\pi_{f}(x)$ defined in (1.5) is to generate the primes up to $x$ by Eratosthenes's sieve, which is too expensive for large values of $x$.

DeFinition 7.1. An arithmetic function $f$ is said to be completely multiplicative if $f(a b)=$ $f(a) f(b)$ for all $a$ and $b$. If $f \neq 0$, this implies $f(1)=1$.

Following ideas of the german astronomer Meissel, Lagarias, Miller and Odlyzko gave in [10] an algorithm that computes $\pi(x)$ with a cost $O\left(x^{2 / 3} / \log x\right)$. In this work they also remark that their algorithm allows to compute $\pi_{f}(x)$ for every completely multiplicative arithmetic function $f$.

This method has been improved in [11] to compute $\pi(x)$ with a cost $O\left(x^{2 / 3} / \log ^{2} x\right)$, provided that all the arithmetic operations on integers are of constant cost $O(1)$, not depending on the size of the operands. We show here that this improved algorithm may be used to compute $\pi_{f}(x)$ whith a cost which is still $O\left(x^{2 / 3} / \log ^{2} x\right)$, for a large subset of the set of completely multiplicative arithmetic functions. More precisely we have the proposition. 
Proposition 7.1. Let $f$ be a completely multiplicative arithmetic function with integer values. Let $F$ be the summatory function of $f$,

$$
F(x)=\sum_{n \leqslant x} f(n)
$$

We suppose that all the ordinary arithmetic operations about integers are of constant cost $O(1)$, and that

- each value $f(n)$ may be computed in time $O(1)$, not depending of the size of $n$;

- there is an algorithm computing

$$
S_{0}(y, x)=\sum_{1 \leqslant n \leqslant y} \mu(n) f(n) F\left(\frac{x}{n}\right)
$$

in time $O\left(x^{2 / 3} / \log ^{2} x\right)$.

Then, there is an algorithm computing

$$
\pi_{f}(x)=\sum_{\substack{p \leqslant x \\ p \text { prime }}} f(p)
$$

in time $O\left(x^{2 / 3} / \log ^{2} x\right)$.

When $F(u)$ can be computed in $O(1)$ time, the second hypothesis is satisfied.

REMARK 7.1. The second hypothesis may seem strange. Let us give a few words of explanation.

Our computation of $\pi_{f}(x)$ begins by choosing $y=O\left(x^{1 / 3+\varepsilon}\right)$. Then we compute $S_{0}=S_{0}(y, x)$ (this is the contribution of ordinary leaves defined in Lemma 5.2, equation (9) in [11] and in Lemma 7.2, equation (7.14) in this article). Function $F$ does not appear elsewhere in the algorithm. $S_{0}$ being computed, the total cost of the other computations is $O\left(x^{2 / 3} / \log ^{2} x\right)$. The second condition of Proposition 7.1 ensures that our algorithm computes $\pi_{f}(x)$ in time $O\left(x^{2 / 3} / \log ^{2} x\right)$.

In many cases, $F(u)$ can be computed in time $O(1)$, then the sum (7.2) can be computed in time $O(y)$, by precomputing the Möbius function, so that the second hypothesis is satisfied.

REMARK 7.2. In Proposition 7.1 we restrict ourseves to the case of integer valued functions. The case of real valued functions is more delicate because of truncation errors. In [12], Bach and al. have elaborated an algorithm to compute $\pi_{f}(x)$ where $f(n)=1 / n$, and

$$
x=1801241484456448000=1.8 \ldots \times 10^{18} .
$$

7.1. Algorithm for $\boldsymbol{\pi}_{\boldsymbol{f}}(\boldsymbol{x})$. We will describe very briefly our algorithm to compute $\pi_{f}(x)$, using notations and formulas which, when replacing $f$ by 1 , reduce to the correponding ones contained in [11].

For $b \in \mathbb{N}$, let us define $\Phi(x, b)$ as the sum of the $f(n)$, for the $n^{\prime} s \in[1, x]$ that subsist after sieving this interval by all primes $p_{1}, p_{2}, \ldots, p_{b}$,

$$
\Phi(x, 0)=\sum_{1 \leqslant n \leqslant x} f(n)=F(x), \quad \Phi(x, b)=\sum_{\substack{1 \leqslant n \leqslant x \\ P^{-}(n)>p_{b}}} f(n), \quad b \geqslant 1 .
$$

For $k \geqslant 1$ and $b \geqslant 1$, let us set

$$
P_{k}(x, b)=\sum_{\substack{1 \leqslant n \leqslant x \\ \Omega(n)=k, P^{-}(n)>p_{b}}} f(n)
$$


so that, from (7.3) and (7.4) we get, for $x \geqslant 1$,

$$
\Phi(x, b)=1+P_{1}(x, b)+P_{2}(x, b)+\cdots .
$$

From now on, we choose $y \in \mathbb{R}$

$$
x^{1 / 3} \leqslant y \leqslant \sqrt{x}
$$

and set

$$
a=\pi(y) .
$$

We will precise later the best choice for $y$, which is closed to $x^{1 / 3}$.

Since $y \geqslant x^{1 / 3}$ equation (7.4) yields $P_{k}(x, a)=0$ for $k \geqslant 3$ and (7.5) becomes

$$
\Phi(x, a)=1+P_{1}(x, a)+P_{2}(x, a) .
$$

Since

$$
P_{1}(x, a)=\sum_{p_{a}<p \leqslant x} f(p)=\sum_{y<p \leqslant x} f(p)=\pi_{f}(x)-\pi_{f}(y),
$$

we have

$$
\pi_{f}(x)=\Phi(x, a)+\pi_{f}(y)-1-P_{2}(x, a) .
$$

Replacing $f$ by 1 (and $\pi_{f}$ by $\pi$ ), formula (7.7) is formula (4) in [11].

7.2. Initialization of the computation: the two basis tables. After fixing $y$, by using Eratosthenes's sieve, we precompute the table of primes up to $y$, and the table of the values $\pi_{f}(u)$ for $1 \leqslant u \leqslant y$. The cost of these initializations is $O(y \log \log y)$.

7.3. Computation of $\boldsymbol{P}_{\mathbf{2}}(\boldsymbol{x}, \boldsymbol{a})$. Definition (7.4) and the complete multiplicativity of $f$ give

$$
P_{2}(x, a)=\sum_{\substack{y<p \leqslant q \leqslant x \\ p q \leqslant x}} f(p q)=\sum_{\substack{y<p \leqslant q \leqslant x \\ p q \leqslant x}} f(p) f(q)
$$

where $p$ and $q$ are primes. The $p^{\prime} s$ figuring in this sum satisfy $p \leqslant x / q \leqslant x / y$ and we get

$$
P_{2}(x, a)=\sum_{y<p \leqslant x / y} f(p) \sum_{p \leqslant q \leqslant x / p} f(q) .
$$

We remark that, for $p>\sqrt{x}$, the sum on $q$ vanishes. Since, by $(7.6), \sqrt{x} \leqslant x / y$, we have

$$
P_{2}(x, a)=\sum_{y<p \leqslant \sqrt{x}} f(p) \sum_{p \leqslant q \leqslant x / p} f(q)=\sum_{y<p \leqslant \sqrt{x}} f(p)\left(\pi_{f}\left(\frac{x}{p}\right)-\pi_{f}(p-1)\right)
$$

or

$$
P_{2}(x, a)=\sum_{y<p \leqslant \sqrt{x}} f(p) \pi_{f}\left(\frac{x}{p}\right)-\sum_{y<p \leqslant \sqrt{x}} f(p) \pi_{f}(p-1) .
$$

In the above formula, the values of $p$ are bounded above by $\sqrt{x}$ which is larger than $y$. Thus we cannot find these primes $p$, nor the values $\pi_{f}(p-1)$, in the precomputed tables (cf. Section 7.2), and we generate them using a sieve of $[1, \sqrt{x}]$, which we call the auxilliary sieve. The values of $x / p$ lie in the interval $[1, x / y]$. So we will get the values $\pi_{f}(x / p)$ by an other sieve, the main sieve. Let us note that the respective sizes of the sieve intervals, $\sqrt{x}$ and $x / y$, are too large to allow a sieve in one pass. Thus the two sieves will be done by blocks of size $y$ that must be synchronized.

Initialization. Computation of $\varpi$, the largest prime $\leqslant \sqrt{x}$ and of $\pi_{f}(\varpi)$. By Eratosthenes'sieve we compute the largest prime $\varpi$ not exceeding $\sqrt{x}$ and calculate $\pi_{f}(\varpi)$. The auxilliary sieve is then initialized by putting in the sieve-table the primes $p$ of the block $[\sqrt{x}-y+1, \sqrt{x}]$. The main 
sieve is initialized by sieving the first block $[A, B]=[\sqrt{x}, \sqrt{x}+y-1]$. The cost of this phase is $O\left(x^{1 / 2} \log \log x\right)$.

Computing $P_{2}(x, a)$. We use formula (7.8), getting in decreasing order the primes $p \in(y, \sqrt{x}]$ and the $f(p) \pi_{f}(p-1)$ from the auxillary sieve, and getting the values $\pi_{f}(x / p)$ from the main sieve whose successive blocks will cover in ascending order the interval $[\sqrt{x}, x / y)$.

We initialize a variable $p$ with the value $\varpi$, a variable $T$ with the value $\pi_{f}(\varpi)$ and a variable $P_{2}$ with the value 0 . Then, while $p>y$, we repeat:

- substract $f(p)$ from $T$; thus the new value of $T$ is $\pi_{f}(p-1)$;

- if $x / p>B$, while $x / p>B$ we replace the block $[A, B]$ by the next block $[A+y, B+y]$ and we sieve it; when $x / p \in[A, B]$ we get $\pi_{f}(x / p)$ in the main sieve table and we add $f(p) \pi_{f}(x / p)-f(p) T$ to $P_{2}$

- using the auxilliary sieve, replace $p$ by its predecessor.

The final value of the variable $P_{2}$ is $P_{2}(x, a)$. The first step is negligible in cost, compared to the second. Thus the computation of $P_{2}(x, a)$ is of total cost

$$
O\left(\frac{x}{y} \log \log y\right)
$$

7.4. Computation of $\boldsymbol{\Phi}(\boldsymbol{x}, \boldsymbol{a})$. The following lemma is proved as Lemma 5.1 in [11].

LEMma 7.1. For every $u \geqslant 0$, and for $b \geqslant 1$,

$$
\begin{gathered}
\Phi(u, 0)=F(u) \\
\Phi(u, b)=\Phi(u, b-1)-f\left(p_{b}\right) \Phi\left(\frac{u}{p_{b}}, b-1\right) .
\end{gathered}
$$

This relation gives an obvious method for computing $\Phi(x, a)$. Starting from the tree with the only node $\Phi(x, a)$, and applying repeatedly (7.10) we get a tree whose all nodes, except the root node, are labelled by a formula of the form

$$
\mu(n) f(n) \Phi\left(\frac{x}{n}, b\right)
$$

where $b \leqslant a-1$ and $n=1$ or $n$ is a squarefree integer with prime factors $q \in\left\{p_{b+1}, \ldots, p_{a}\right\}$.

If we repeat this expansion until all the leaves of the resulting tree are labelled by formulas $\mu(n) f(n) \Phi(x / n, 0)$, using (7.9), we get the formula:

$$
\Phi(x, a)=\sum_{\substack{1 \leqslant n \leqslant x \\ P^{+}(n) \leqslant y}} \mu(n) f(n) \Phi\left(\frac{x}{n}, 0\right)=\sum_{\substack{1 \leqslant n \leqslant x \\ P^{+}(n) \leqslant y}} \mu(n) f(n) F\left(\frac{x}{n}\right)
$$

which, when $f=1$ is formula

$$
\Phi(x, a)=\sum_{\substack{1 \leqslant n \leqslant x \\ P^{+}(n) \leqslant y}} \mu(n)\left\lfloor\frac{x}{n}\right\rfloor
$$

(cf. [11; p. 237]).

The number of terms in (7.12) is much too large. In order to get a sum with fewer terms we replace the trivial rule

RulE 1. Expand (7.11) using (7.10) if $b>0$, by the new rule

Rule 2. Expand node (7.11) only if $b>0$ and $n \leqslant y$. 
Expanding the computation tree with Rule 2 instead of Rule 1 we get

LEMMA 7.2. We have

$$
\Phi(x, b)=S_{0}+S
$$

where $S_{0}$ is the contribution of ordinary leaves

$$
S_{0}=S_{0}(y, x)=\sum_{1 \leqslant n \leqslant y} \mu(n) f(n) \Phi\left(\frac{x}{n}, 0\right)=\sum_{1 \leqslant n \leqslant y} \mu(n) f(n) F\left(\frac{x}{n}\right)
$$

and $S$, the contribution of special leaves, is

$$
S=\sum_{n / P^{-}(n) \leqslant y<n} \mu(n) f(n) \Phi\left(\frac{x}{n}, \pi\left(P^{-}(n)\right)-1\right) .
$$

This lemma corresponds to Lemma 5.2 in [11].

7.4.1. Computation of $S_{0}$. In the general case, the computation of $S_{0}$ is done with a cost $O\left(x^{2 / 3} / \log ^{2} x\right)$ thanks to the condition 2 in Proposition 7.1.

In the case we will consider later in this work, the computation of $\pi_{\text {id }}(x), f(n)=n$, thus $F(u)=[u][u+1] / 2$ is computed in $O(1)$ time and the computation of $S_{0}(x, y)$ is of cost $O(y)=$ $o\left(x^{2 / 3} / \log ^{2} x\right)$.

7.4.2. Computation of $S$. In the sum (7.13), let us set $n=m p$ with $p=P^{-}(n)$. Grouping together all the $n$ 's according to the value of $p$, we get

$$
S=-\sum_{p \leqslant y} f(p) \sum_{\substack{P^{-}(m)>p \\ m \leqslant y<m p}} \mu(m) f(m) \Phi\left(\frac{x}{m p}, \pi(p)-1\right) .
$$

The computation of $S$ from (7.14) is the complicate part of the algorithm. In the following paragraph we show that it is relatively simple to get a cost $O\left(x^{2 / 3+\varepsilon}\right)$.

7.4.3. How to compute $S$ in $O\left(x^{2 / 3+\varepsilon}\right)$. In this section, we explain a first method to get $\pi_{f}(x)$, rather simple to implement, and whose running time is $O\left(x^{2 / 3+\varepsilon}\right)$. We take $y=x^{1 / 3}$. Since $m p>y$ all the values $u=x /(m p)$ appearing in (7.14) are less than $x^{2 / 3}$. We sieve the interval $[1, x / y)$ successively by all primes $p \leqslant y$. After the sieve by $p$, from the definition (7.3) of $\Phi$, for all the $m$ 's such that $m \leqslant y<m p$, we get in the sieve table the value $\Phi(x /(m p), \pi(p)-1)$, and we add to $S$ the value $f(p) \mu(m) f(m) \Phi(x /(m p), \pi(p)-1)$.

But, if we proceed in the naive way, after sieving by each $p$, we will update the sieve table, putting in the case of index $u$ the sum of $f(n)$ for the $n$ 's, $n \leqslant u$ that are still in the table. This is excluded because, for each $p$ this would cost $O(x / y)$ operations, and the total cost of these updatings would be $\gg \pi(y)(x / y) \asymp x / \log x$. As explained in [10] (the 7 last-lines p. 545 and the first half of p. 546) we use an auxiliary data structure such that, for a price of $O(\log x)$ time in place of $O(1)$ for each access, we don't need to update the sieve table after each sieve. To be a little more precise let us say that this structure is a labelled binary tree. There is a leave for each index $i$ of the table sieve, this leave is labelled by the value $f(i)$, and each interior node is labelled by the sum of labels of its two sons. Proceeding in this way, the cost of the sieve is $O((x / y) \log x \log \log x)$, while the cost of retrieving the values $f(p) \mu(m) f(m) \Phi(x /(m p), \pi(p)-1)$ is $O(\pi(y) y \log x)$. Both costs are $O\left(x^{2 / 3+\varepsilon}\right)$ with our choice $y=x^{1 / 3}$. 
7.4.4. Faster computation of $S$. In this section, we explain how to carry out the computation of $\pi_{f}(x)$ in $O\left(x^{2 / 3} / \log ^{2} x\right)$. We take $y=x^{1 / 3}(\log x)^{3} \log \log x$. To speed up the computation of $S$ we partition (7.14) in tree subsums $S=S_{1}+S_{2}+S_{3}$,

$$
\begin{aligned}
S_{1} & =-\sum_{x^{1 / 3}<p \leqslant y} f(p) \sum_{\substack{P^{-}(m)>p \\
m \leqslant y<m p}} \mu(m) f(m) \Phi\left(\frac{x}{m p}, \pi(p)-1\right), \\
S_{2} & =-\sum_{x^{1 / 4}<p \leqslant x^{1 / 3}} f(p) \sum_{\substack{P^{-}(m)>p \\
m \leqslant y<m p}} \mu(m) f(m) \Phi\left(\frac{x}{m p}, \pi(p)-1\right), \\
S_{3} & =-\sum_{p \leqslant x^{1 / 4}} f(p) \sum_{\substack{P^{-}(m)>p \\
m \leqslant y<m p}} \mu(m) f(m) \Phi\left(\frac{x}{m p}, \pi(p)-1\right) .
\end{aligned}
$$

We will show that $S_{1}$ is quickly computed in $O(y)$ time. $S_{3}$ will be computed by sieve, as explained in Section 7.4.3, but faster, because the number of values for $p$ is reduced from $\pi(y)$ to $\pi\left(x^{1 / 4}\right)$. The main part of the computation will be the computation of $S_{2}$.

As in [11], we first observe that the $m$ 's involved in $S_{1}$ and $S_{2}$ are all prime and therefore:

$$
\begin{aligned}
S_{1} & =\sum_{x^{1 / 3}<p \leqslant y} f(p) \sum_{p<q \leqslant y} f(q) \Phi\left(\frac{x}{p q}, \pi(p)-1\right), \\
S_{2} & =\sum_{x^{1 / 4}<p \leqslant x^{1 / 3}} f(p) \sum_{p<q \leqslant y} f(q) \Phi\left(\frac{x}{p q}, \pi(p)-1\right) .
\end{aligned}
$$

Computing $S_{1}$. As in [11] we remark that, in (7.15), we have $x /(p q)<x^{1 / 3}<p$. Thus, all the values $\Phi(x /(p q), \pi(p)-1)$ are equal to 1 . Therefore

$$
S_{1}=\sum_{x^{1 / 3}<p \leqslant y} f(p) \sum_{p<q \leqslant y} f(q)=\sum_{x^{1 / 3}<p \leqslant y} f(p)\left(\pi_{f}(y)-\pi_{f}(p)\right) .
$$

This value is computed in $O(y)$ additions, using the precomputed table of the values $\pi_{f}(u)$ for $1 \leqslant u \leqslant y$.

Computing $S_{3}$. For each $p \leqslant x^{1 / 4}$ we precompute the list of all the squarefree $m \leqslant y$ whose least factor is $p$.

We sieve the interval $[1, x / y]$ successively by all the primes up to $x^{1 / 4}$. As soon as we have sieved by $p$, using the precomputded lists of squarefree whose least prime factor is a prime $q>p$ we sum

$$
f(p) \sum_{\substack{P^{-}(m)>p \\ m \leqslant y<m p}} \mu(m) f(m) \Phi\left(\frac{x}{m p}, \pi(p)-1\right)
$$

for all squarefree $m \in[y / p, y)$ such that $P^{-}(m)>p$. This computation is done by blocks, using the auxiliary structure, as explained at the end of Section 7.4.3.

Thus the cost of sieving is $O((x / y) \log x \log \log x)$. The number of values of $p$ is $\pi\left(x^{1 / 4}\right)$ and the number of values of $m$ is less than $y$, thus the cost of retrieving the values $f(p) \mu(m) f(m) \Phi(x /(m p)$, $\pi(p)-1)$ is $O\left(\pi\left(x^{1 / 4}\right) \times \log x \times y\right)$ Thus computing $S_{3}$ is of cost $O\left((x / y) \log x \log \log x+y x^{1 / 4}\right)$.

Computing $S_{2}$. We split the sum (7.16) in two parts depending on $q>x / p^{2}$ or $q \leqslant x / p^{2}$. It gives

$$
S_{2}=U+V
$$


with

$$
U=\sum_{x^{1 / 4}<p \leqslant x^{1 / 3}} f(p) \sum_{\substack{p<q \leqslant y \\ q>x / p^{2}}} f(q) \Phi\left(\frac{x}{p q}, \pi(p)-1\right)
$$

and

$$
V=\sum_{x^{1 / 4}<p \leqslant x^{1 / 3}} f(p) \sum_{\substack{p<q \leqslant y \\ q \leqslant x / p^{2}}} f(q) \Phi\left(\frac{x}{p q}, \pi(p)-1\right)
$$

Computing $U$. With $y<\sqrt{x}$ (cf. (7.6)), the condition $q>x / p^{2}$ implies $p^{2}>x / q \geqslant x / y \geqslant x^{1 / 2}$. Thus,

$$
U=\sum_{\sqrt{x / y}<p \leqslant x^{1 / 3}} f(p) \sum_{\substack{p<q \leqslant y \\ q>x / p^{2}}} f(q) \Phi\left(\frac{x}{p q}, \pi(p)-1\right)
$$

From $x / p^{2}<q$ we deduce $x /(p q)<p$ and $\Phi(x /(p q), \pi(p)-1)=1$, and we have $x / p^{2} \geqslant p$ so that

$$
U=\sum_{\sqrt{x / y}<p \leqslant x^{1 / 3}} f(p) \sum_{\substack{p<q \leqslant y \\ q>x / p^{2}}} f(q)=\sum_{\sqrt{x / y}<p \leqslant x^{1 / 3}} f(p)\left(\pi_{f}(y)-\pi_{f}\left(\frac{x}{p^{2}}\right)\right)
$$

Since $x / p^{2}<q \leqslant y$ the sum $U$ is calculated in $O(y)$ operations with the table of values of $\pi_{f}(u)$.

Computing $V$. For each term involved in $V$ we have $p \leqslant x /(p q)<x^{1 / 2}<p^{2}$. Hence, by (7.3), $\Phi(x /(p q), \pi(p)-1)$ is the sum of $f(n)$ for $n$ satisfying $n \leqslant x /(p q)$ and $P^{-}(n) \geqslant p$. These $n$ 's are $n=1$ and all the primes $n$ satisfying $p-1<n \leqslant x /(p q)$. Thus

$$
\Phi\left(\frac{x}{p q}, \pi(p)-1\right)=1+\pi_{f}\left(\frac{x}{p q}\right)-\pi_{f}(p-1) .
$$

And we write

$$
V=V_{1}+V_{2}
$$

with

$$
\begin{aligned}
& V_{1}=\sum_{x^{1 / 4} \leqslant p<x^{1 / 3}} f(p) \sum_{p<q \leqslant \min \left(x / p^{2}, y\right)} f(q)\left(1-\pi_{f}(p-1)\right), \\
& V_{2}=\sum_{x^{1 / 4} \leqslant p<x^{1 / 3}} f(p) \sum_{p<q \leqslant \min \left(x / p^{2}, y\right)} f(q) \pi_{f}\left(\frac{x}{p q}\right) .
\end{aligned}
$$

Computing $V_{1}$ can be achieved in $O(y)$ time once we have tabulated $\pi_{f}(u)$ for $u \leqslant y$.

Computing $V_{2}$. We first split $V_{2}$ in two parts in order to simplify the condition $q \leqslant \min \left(x / p^{2}, y\right)$ :

$$
V_{2}=\sum_{x^{1 / 4}<p \leqslant \sqrt{x / y}} f(p) \sum_{p<q \leqslant y} f(q) \pi_{f}\left(\frac{x}{p q}\right)+\sum_{\sqrt{x / y}<p<x^{1 / 3}} f(p) \sum_{p<q \leqslant x / p^{2}} f(q) \pi_{f}\left(\frac{x}{p q}\right) .
$$


In the purpose to speed up the computation of the above two sums we now write, $V_{2}=W_{1}+W_{2}+$ $W_{3}+W_{4}+W_{5}$ with

$$
\begin{aligned}
W_{1}= & \sum_{x^{1 / 4}<p \leqslant x / y^{2}} f(p) \sum_{p<q \leqslant y} f(q) \pi_{f}\left(\frac{x}{p q}\right), \\
W_{2}= & \sum_{x / y^{2}<p \leqslant \sqrt{x / y}} f(p) \sum_{p<q \leqslant \sqrt{x / p}} f(q) \pi_{f}\left(\frac{x}{p q}\right), \\
W_{3}= & \sum_{x / y^{2}<p \leqslant \sqrt{x / y}} f(p) \sum_{\sqrt{x / p}<q \leqslant y} f(q) \pi_{f}\left(\frac{x}{p q}\right), \\
W_{4}= & \sum_{\sqrt{x / y}<p \leqslant x^{1 / 3}} f(p) \sum_{p<q \leqslant \sqrt{x / p}} f(q) \pi_{f}\left(\frac{x}{p q}\right), \\
W_{5}= & \sum_{\sqrt{x / y}<p \leqslant x^{1 / 3}} f(p) \sum_{\sqrt{x / p}<q \leqslant x / p^{2}} f(q) \pi_{f}\left(\frac{x}{p q}\right) .
\end{aligned}
$$

Computing $W_{1}$ and $W_{2}$. These two quantities need values of $\pi_{f}(x /(p q))$ with $x^{1 / 3}<x /(p q)<$ $x^{1 / 2}$. These are computed with a sieve of the interval $[1, \sqrt{x}]$. The sieving is done by blocks, and, for each block, we sum $f(p) f(q) \pi_{f}(x /(p q))$ for the pairs $(p, q)$ satisfying the conditions of the sum $W_{1}$ or $W_{2}$ and such that $x /(p q)$ lies in the block.

The cost of this computation is the sum of three terms:

- the cost of the above sieve on $[1, \sqrt{x}]$ is $O(\sqrt{x} \log \log x)$;

- the cost of adding the terms of the sum $W_{1}, O\left(x /\left(y \log ^{2} x\right)\right)$;

- the cost of adding the terms of the sum $W_{2}, O\left(x^{3 / 4} /\left(y^{1 / 4} \log ^{2} x\right)\right)$.

Computing $W_{3}$ and $W_{5}$. For $W_{3}$, for each $p$ we apply Lemma 2.6 with $z=x / p$ and $u=y$. Thus, for each value of $p$, the sum on $q \operatorname{costs} O(\pi(\sqrt{x / p}))$, and the total cost of the computation of $W_{3}$ is

$$
O\left(\sum_{x / y^{2}<p \leqslant x / y} \pi\left(\sqrt{\frac{x}{p}}\right)\right) .
$$

For $W_{5}$, for each $p$ we apply lemma 2.6 with $z=x / p$ and $u=x / p^{2}$. Thus, for each value of $p$, the sum on $q$ costs $O(\pi(\sqrt{x / p}))$, and the total cost of the computation of $W_{5}$ is

$$
O\left(\sum_{\sqrt{x / y}<p \leqslant x^{1 / 3}} \pi\left(\sqrt{\frac{x}{p}}\right)\right) .
$$

Thus the costs of computing $W_{3}$ and $W_{5}$ add to

$$
O\left(\sum_{x / y^{2}<p \leqslant x^{1 / 3}} \pi\left(\sqrt{\frac{x}{p}}\right)\right)=O\left(\sum_{x / y^{2}<p \leqslant x^{1 / 3}}\left(\frac{\sqrt{x / p}}{\log (x / p)}\right)\right)=O\left(\frac{x^{2 / 3}}{\log ^{2} x}\right) .
$$

Computing $W_{4}$. We simply sum over $(p, q)$. There would be no advantage to proceed as for $W_{3}$ since most of the values $\pi_{f}(x /(p q))$ are distinct. The cost is

$$
O\left(\sum_{\sqrt{x / y}<p \leqslant x^{1 / 3}} \pi\left(\sqrt{\frac{x}{p}}\right)\right)=O\left(\frac{x^{2 / 3}}{\log ^{2} x}\right) .
$$

As in [11; Section 8], we then see that, since $y=x^{1 / 3} \log ^{3} x \log \log x$, the total cost of the computation of $\pi_{f}(x)$ is $O\left(x^{2 / 3} / \log ^{2} x\right)$. 


\section{The algorithm to calculate $h(n)$}

8.1. The function $\boldsymbol{G}\left(\boldsymbol{p}_{\boldsymbol{k}}, \boldsymbol{m}\right)$. The function $G\left(p_{k}, m\right)$ has been introduced and studied in [5].

DeFinition 8.1. Let $p_{k}$ be the $k$ th prime, for some $k \geqslant 3$ and $m$ an integer satisfying $0 \leqslant m \leqslant$ $p_{k+1}-3$. We define

$$
G\left(p_{k}, m\right)=\max \frac{Q_{1} Q_{2} \cdots Q_{s}}{q_{1} q_{2} \cdots q_{s}}
$$

where the maximum is taken over the primes $Q_{1}, Q_{2}, \ldots, Q_{s}, q_{1}, q_{2}, \ldots, q_{s}, s \geqslant 0$, satisfying

$$
3 \leqslant q_{s}<q_{s-1}<\cdots<q_{1} \leqslant p_{k}<p_{k+1} \leqslant Q_{1}<Q_{2}<\cdots<Q_{s}
$$

and

$$
\sum_{i=1}^{s}\left(Q_{i}-q_{i}\right) \leqslant m
$$

The additive function $\ell$ (cf. Section 1.1) can easily be extended to fractions by setting

$$
\ell\left(\frac{M}{N}\right)=\ell(M)-\ell(N)
$$

when $M$ and $N$ are coprime or are both squarefree. Therefore, the inequality (8.3) implies

$$
\ell\left(G\left(p_{k}, m\right)\right) \leqslant m .
$$

8.1.1. Properties of $G\left(p_{k}, m\right)$. Obviously, $G\left(p_{k}, m\right)$ is non-decreasing on $m$ and $G\left(p_{k}, 2 m+1\right)=$ $G\left(p_{k}, 2 m\right)$. The maximum in (8.1) is unique (from the uniqueness of the standard factorization into primes). For small $m$ 's, we have

$$
0 \leqslant m<p_{k+1}-p_{k} \quad \Longrightarrow \quad G\left(p_{k}, m\right)=1 .
$$

From Proposition 8 of [5], we have

$$
\frac{p_{k+1}}{\left(p_{k+1}-m\right)^{\star}} \leqslant G\left(p_{k}, m\right) \leqslant \frac{p_{k+1}}{p_{k+1}-m} .
$$

Note that if $p_{k+1}-m$ is prime, then (8.6) yields the exact value of $G\left(p_{k}, m\right)$.

8.1.2. Computation of $G\left(p_{k}, m\right)$. In [5; Section 8], two algorithms are given to calculate $G\left(p_{k}, m\right)$.

The first one is a combinatorial algorithm. In its first step, the primes allowed to divide the denominator of $G\left(p_{k}, m\right)$ are determined. From (8.2) and (8.3), they are all the primes in the range $\left[\left(p_{k+1}-m\right), p_{k}\right]$, say $P_{1}<P_{2}<\cdots<P_{K}$. Similarly, the primes authorized to divide the numerator are all the primes $P_{K+1}<P_{K+2}<\cdots<P_{R}$ in $\left[p_{k+1}, p_{k}+m\right]$. By setting $\mathcal{P}^{\prime}=\left\{P_{1}, P_{2}, \ldots, P_{R}\right\}$, from the definition (1.14), we get

$$
G\left(p_{k}, m\right)=\frac{1}{P_{1} P_{2} \cdots P_{K}} h_{K}\left(P_{1}+P_{2}+\cdots+P_{K}+m, \mathcal{P}^{\prime}\right)
$$

and $h_{j}\left(n, \mathcal{P}^{\prime}\right)$ can be computed by induction on $j$ in a way similar to that exposed in Section 1.4. In [5; Section 8], one can find the details and also some tricks to improve the running time of this combinatorial algorithm which, however, remains rather slow when $m$ is large.

The second algorithm, which is more sophisticated, is based on the following remark: if $G\left(p_{k}, m\right)=Q_{1} Q_{2} \cdots Q_{s} /\left(q_{1} q_{2} \cdots q_{s}\right)$ and $m$ is large, the least prime factor $q_{s}$ of the denominator is close to $p_{k+1}-m$ while all the other primes $Q_{1}, \ldots, Q_{s}, q_{1}, \ldots, q_{s-1}$ are close to $p_{k}$.

More precisely, the following proposition (which is Proposition 10 of [5]) says that if $p_{k+1}-m+\delta$ is prime for some small $\delta$ and if $G\left(p_{k+1}, \delta\right)$ is not too small, then the computation of $G\left(p_{k}, m\right)$ is reduced to the computation of $G\left(p_{k+1}, m^{\prime}\right)$ for few small values of $m^{\prime}$, which can be done by the above combinatorial algorithm. 
Proposition 8.1. We want to compute $G\left(p_{k}, m\right)$ as defined in (8.1) with $p_{k}$ odd and $p_{k+1}-p_{k} \leqslant$ $m \leqslant p_{k+1}-3$. We assume that we know some even non-negative integer $\delta$ satisfying

$$
\begin{gathered}
p_{k+1}-m+\delta \quad \text { is prime } \\
G\left(p_{k+1}, \delta\right) \geqslant 1+\frac{\delta}{p_{k+1}}
\end{gathered}
$$

and

$$
\delta<\frac{2 m}{9}<\frac{2 p_{k+1}}{9} .
$$

If $\delta=0$, we know from (8.6) that $G\left(p_{k}, m\right)=p_{k+1} /\left(p_{k+1}-m\right)$. If $\delta>0$, we have

$$
G\left(p_{k}, m\right)=\max _{\substack{q \text { prime } \\ p_{k+1}-m \leqslant q \leqslant \widehat{q}}} \frac{p_{k+1}}{q} G\left(p_{k+1}, m-p_{k+1}+q\right),
$$

where $\widehat{q}$ is defined by

$$
\widehat{q}=\frac{p_{k+1} p_{k+2}\left(p_{k+1}-m+\delta\right)}{\left(p_{k+1}+\delta\right)\left(p_{k+1}-3 \delta / 2\right)} \leqslant p_{k+2}-m+\frac{3 \delta}{2} .
$$

How to compute $G\left(p_{k}, m\right)$ ? The combinatorial algorithm should be tried if $m$ is small, but it is quadratic in $m$ and has no chance to terminate if $m$ is larger than, say, $10^{6}$. We have no guarantee that the conditions of Proposition 8.1 are satisfied. However in all our numerical applications, we have found $\delta<1000$ in (8.7) (see [5; Section 9.2]), so that, by (8.10) and (8.11), we have

$$
m-p_{k+1}+q \leqslant m-p_{k+1}+\widehat{q} \leqslant p_{k+2}-p_{k+1}+\frac{3 \delta}{2}
$$

and, in (8.10), $G\left(p_{k+1}, m-p_{k+1}+q\right)$ can be easily calculated by the combinatorial algorithm.

8.2. Description of the algorithm to compute $\boldsymbol{h}(\boldsymbol{n})$. To compute $h(n)$, the first step is to determine $p_{k}$ and $\sigma_{k}$ defined by (1.8). This step is explained in Section 8.2.1 and will furnish also $p_{k+1}$ and $n^{\prime}=n-p_{k}$.

8.2.1. Computation of $p_{k}$ and $\sigma_{k}$. Compute $x=\sqrt{\mathrm{Li}^{-1}(n)}$, so that $\operatorname{Li}\left(x^{2}\right)=n$ and $x \sim$ $\sqrt{n \log n}$.

Using Proposition 7.1, we compute $\pi_{\text {id }}(x)$ in time $O\left(x^{2 / 3} / \log ^{2} x\right)=O\left(n^{1 / 3} /(\log n)^{-5 / 3}\right)$.

To get $\sigma_{k}$, we have to add (if $\pi_{\mathrm{id}}(x)<n$ ) or to subtract (if $\pi_{\mathrm{id}}(x)>n$ ) to $\pi_{\mathrm{id}}(x)$ the primes between $x$ and $p_{k}$, calculated by sieving. In practice, this step is very short. But we are able to give a small estimate for it only under Riemann's hypothesis. By Lemma 2.5, we have

$$
\operatorname{Li}\left(p_{k}^{2}\right)-\frac{5}{24 \pi} p_{k}^{3 / 2} \log p_{k}<\sigma_{k} \leqslant n<\sigma_{k+1}<\operatorname{Li}\left(p_{k+1}^{2}\right)+\frac{5}{24 \pi} p_{k+1}^{3 / 2} \log p_{k+1}
$$

which implies

$$
n=\operatorname{Li}\left(p_{k}^{2}\right)+O\left(p_{k}^{3 / 2} \log p_{k}\right) \sim \operatorname{Li}\left(p_{k}^{2}\right) \sim \frac{p_{k}^{2}}{2 \log p_{k}} .
$$

Therefore, we get $\log n \sim 2 \log p_{k}, p_{k} \sim \sqrt{n \log n}$ and

$$
\operatorname{Li}\left(p_{k}^{2}\right)=n+O\left(n^{3 / 4}(\log n)^{7 / 4}\right) .
$$

Further, since $x \sim p_{k} \sim \sqrt{n \log n}$, we have

$$
\left|n-\operatorname{Li}\left(p_{k}^{2}\right)\right|=\left|\operatorname{Li}\left(x^{2}\right)-\operatorname{Li}\left(p_{k}^{2}\right)\right| \sim \frac{\left|x^{2}-p_{k}^{2}\right|}{2 \log x} \sim 2\left|x-p_{k}\right| \sqrt{\frac{n}{\log n}},
$$

so that, from (8.12),

$$
\left|\pi(x)-\pi\left(p_{k}\right)\right| \leqslant\left|x-p_{k}\right|=O\left(n^{1 / 4}(\log n)^{9 / 4}\right) .
$$


8.2.2. Computation of $h(n) / N_{k}$. By Corollary 6.1, we have $h(n)=h_{k}(n)$. Let us set $n^{\prime}=n-\sigma_{k}$. If $n^{\prime}=p_{k+1}-1$ or $n^{\prime}=p_{k+1}-2$, Proposition 5.3 yields $h(n)=N_{k+1} / 2$. So, we may suppose $n^{\prime} \leqslant \sigma_{k+1}-3$. From the definition (8.1) of function $G$, we have

$$
h(n)=h_{k}(n)=N_{k} G\left(p_{k}, n^{\prime}\right)
$$

and we compute $G\left(p_{k}, n^{\prime}\right)$ as explained in Section 8.1.2. In practice, the computation of $G\left(p_{k}, n\right)$ is very fast. However, as explained in Section 8.1.2, we have no estimation of the running time.

Below, are listed some values of $h(n) / N_{k}=G\left(p_{k}, n^{\prime}\right)$ together with $p_{k}, n^{\prime}=n-\sigma_{k}, e=e(n)$ the largest integer such that $h(n-e)=h(n)$ and, if the algorithm of Proposition 8.1 is used, $\delta$ and $Q$, the number of primes used in the sum (8.10),

$$
\begin{gathered}
n=10^{12}, \quad p_{k}=5477081, \quad n^{\prime}=4935150, \quad e=0, \quad \delta=18, \quad Q=1, \\
G\left(p_{k}, n^{\prime}\right)=\frac{29998525822277}{2968309525031}=\frac{5477089 \cdot 5477093}{5477081 \cdot 541951} ; \\
n=10^{35}, \quad p_{k}=2898434150644708999, \quad n^{\prime}=1886081812111845520, \quad e=16, \\
\delta=134, \quad Q=5, \quad G\left(p_{k}, n^{\prime}\right)=\frac{2898434150644709023}{1012352338532863519} .
\end{gathered}
$$

The values of $h\left(10^{a}\right)$ for $a \leqslant 35$ and of $h=2^{b}$ for $b \leqslant 116$ can be found on the authors's web sites [2], [3], together with the Maple or Sage programs computing $h(n) / N_{k}$.

\section{An open question}

Given $n$ and $j<k(n)$, how to compute $h_{j}(n)$ ? We have not succeeded in solving this problem when $n$ is too large to use the naive algorithm described in Section 1.4. The case $j=2$ is already not that simple.

A first step is certainly to calculate $r=r(n, j)$ defined by (4.1), which can be done by the method of Section 8.2.1. If we are lucky enough that $q=p_{j+r+1}-n^{\prime}=p_{j+r+1}-\left(n-\sigma_{j+r}+\sigma_{r}\right)$ is prime, then the value $h_{j}(n)=N_{j+r+1} /\left(q N_{r}\right)$ is given by (4.24).

In the general case, by setting $n^{\prime}=n-\left(\sigma_{j+r}-\sigma_{r}\right)$, one may think that $h_{j}^{\prime}(n)=\left(N_{j+r} / N_{r}\right) \times$ $G\left(p_{j+r}, n^{\prime}\right)$ has a good chance to be the value of $h_{j}(n)$. However, there are exceptions.

\section{Bibliography}

[1] N. J. A. Sloane, S. Plouffe, The Encyclopedia of Integer Sequences, Academic Press, San Diego, CA, 1995; http://oeis.org/.

[2] M. Deléglise, http://math.univ-lyon1.fr/ $\operatorname{deleglis/calculs.html.~}$

[3] J.-L. Nicolas, http://math.univ-lyon1.fr/ nicolas/hden.html.

[4] E. Landau, "Über die Maximalordnung der Permutationen gegebenen Grades", Arch. der Math. und Phys. (3), 5 (1903), 92-103; Handbuch der Lehre von der Verteilung der Primzahlen, Band 1, Chelsea Publ., New York, 1953, 222-229.

[5] M. Deléglise, J.-L. Nicolas, P. Zimmermann, "Landau's function for one million billions", J. Théor. Nombres Bordeaux, 20:3 (2008), 625-671.

[6] M. Deléglise, J.-L. Nicolas, "Le plus grand facteur premier de la fonction de Landau", Ramanujan J., 27:1 (2012), 109-145.

[7] P. Dusart, Estimates of Some Functions Over Primes without R.H., arXiv: 1002.0442v1.

[8] P. Dusart, "The $k$ th prime is greater than $k(\ln k+\ln \ln k-1)$ for $k \geqslant 2$ ", Math. Comp., 68:225 (1999), 411-415. 
[9] L. Schoenfeld, "Sharper bounds for the Chebyshev functions $\theta(x)$ and $\psi(x)$. II", Math. Comp., 30:134 (1976), 337-360.

[10] J. C. Lagarias, V.S. Miller, A.M. Odlyzko, "Computing $\pi(x)$ : the Meissel-Lehmer method", Math. Comp., 44:170 (1985), 537-560.

[11] M. Deléglise, J. Rivat, "Computing $\pi(x)$ : the Meissel, Lehmer, Lagarias, Miller, Odlyzko method", Math. Comp., 65:213 (1996), 235-245.

[12] E. Bach, D. Klyve, J. P. Sorenson, "Computing prime harmonic sums", Math. Comp., 78:268 (2009), $2283-2305$. 